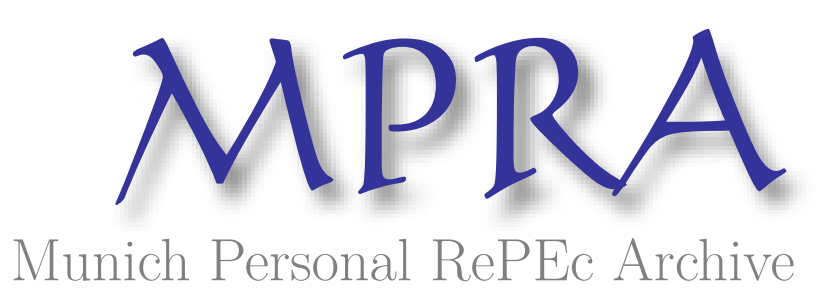

\title{
Language education and economic outcomes in a bilingual society
}

Yuki, Kazuhiro

February 2021

Online at https://mpra.ub.uni-muenchen.de/106119/

MPRA Paper No. 106119, posted 17 Feb 2021 14:48 UTC 


\title{
Language education and economic outcomes in a bilingual society
}

\author{
Kazuhiro Yuki*
}

February 2021

\begin{abstract}
Poor economic performance of minority groups and large economic disparity between these groups and the majority group are major concerns in most countries. In many of these countries, the mother tongue of the latter group is the common language in national business and in inter-group communications. How much weights should be placed on common language education and on ethnic language education is a crucial issue in school education of minority students.

This paper develops a model to examine the issue theoretically. It is shown that balanced education of the two languages is critical for skill development of individuals with limited wealth. It is also found that balanced dual education is desirable in terms of earnings net of educational expenditure and consumption, only when the country has favorable conditions (TFP is reasonably high and education is reasonably effective in skill development) and only for those with adequate wealth. Common-language-only education maximizes net earnings and consumption of those with little wealth, and, when the country's conditions are not good, maximizes the economic outcomes of all. Policy implications of the results are discussed. The paper also examines implications of the asymmetric language positions of the groups for sectoral choices and within-group inequalities.

Keywords: language policy, bilingual education, ethnic inequality, human capital, economic development

JEL classification numbers: I25, J15, J24, O15, Z13
\end{abstract}

*Faculty of Economics, Kyoto University, Yoshida-hommachi, Sakyo-ku, Kyoto, 606-8501, Japan; Phone +81-75753-3532; E-mail yuki@econ.kyoto-u.ac.jp. Valuable comments of seminar participants at Osaka City University are gratefully acknowledged. 


\section{Introduction}

Poor economic performance of minority (or subordinate) groups and large disparity between these groups and the majority (or dominant) group are major concerns in many countries. Large ethnic inequality has serious consequences: Alesina, Michalopoulos, and Papaioannou (2016), based on cross-sectional data of 173 countries, find that inequality in economic well-being among ethnic groups, rather than ethnic diversity per se, is strongly negatively related to economic development.

Discrimination, inequality in endowment such as wealth, and inequality in political power are considered as main factors behind the worrying situation of minority (or subordinate) groups. What receives limited attention is language barriers they face: in many countries, the mother tongue of the largest or most powerful ethnic group is the common language in national business and in inter-group communications, such as Spanish in Bolivia, Mandarin in China, Amharic in Ethiopia, Turkish in Turkey, and Vietnamese in Vietnam.

How much weights should be placed on teaching a local ethnic language and teaching the common language and which language should be used as a language of instruction of other subjects are crucial issues in school education of minority students. Even today, ethnic language education remains limited in many countries: as many as $40 \%$ of students in the world do not have access to education in a language they speak or understand (Walter and Benson, 2012). Recently, countries such as Bolivia (Haboud and Limerick, 2017), Ethiopia (Benson et al., 2012) and the Philippines (Tupas and Martin, 2017) have greatly increased the weight on mother tongue education, while countries such as China (Gao and Wang, 2017), Turkey (Faltis, 2014), and Vietnam (Nguyen and Nguyen, 2019) have maintained or intensified the emphasis on common language education.

Empirical works suggest that acquiring common language skill, though difficult for the minority, is rewarding. Azam, Chin, and Prakash (2013) show that the return to speak fluent English in India is as large as the return to secondary education and half as large as the return to college education, after controlling for education, age, social group, geography, and proxies for ability. Nguyen et al. (2017) find that a lack of proficiency in Vietnamese of the minority is a major contributor to the disparity in household expenditures between them and the majority. By contrast, acquiring the skill to use the ethnic language is less demanding, because it is the mother tongue and a part of the skill is taught at home, but its use is limited to the local ethnic business and community.

A general consensus among specialists on language and education is that placing emphasis on ethnic language education at least in primary education is important for students to acquire adequate language and non-language skill (Ball, 2011; Heugh, 2011). By contrast, we know very little what is a desirable combination of the two types of education in terms of future earnings and consumption and what kind of educational and economic policies should be conducted when both educational and economic outcomes of students are taken into account. The main purpose of this paper is to develop a simple model to examine these important issues theoretically. The paper also examines implications of the fact that the common language is the mother tongue of the majority but not of the minority for sectoral choices and within-group inequalities of the two groups.

Model: The model economy is populated by two ethnic groups, the majority or dominant group (thereafter the majority) and the minority or subordinate group (thereafter the minority), and is composed of three production sectors, the national sector and group-specific local sectors. In the real economy, national (local) sector jobs correspond to many jobs in companies doing business nationwide (locally) and jobs involving communications with other groups (locals).

Working in the national sector requires the skill to use the common language, which is the ethnic language of the majority, while working in the local sector of an ethnic group demands the skill to use the group's ethnic language. This implies that the skills required in the two sectors are 
the same for the majority, while they are different for the minority.

Each person has wealth (endowment) to expend on education for developing these skills. The minority, who build up both skills, cannot choose the allocation of the expenditure over the development of the two skills; it is fixed reflecting the fact that weights on common language education and ethnic language education are mostly determined by the government in basic education. The level of common language skill of the minority is zero without education, while the level of mother tongue skill is positive without education (i.e., a portion of the skill is developed at home). ${ }^{1}$

Individuals must self-finance education due to the absence of credit market. The distribution of wealth is such that some individuals do not have enough wealth to make the optimal level of investment, although the case in which nobody faces the wealth constraint is also analyzed. The setting reflects the fact that, in many countries, students must rely on family wealth to pay for study materials, commuting cost, and others even when public schools do not charge tuitions. After educational investment, individuals choose a sector to work, receive earnings, and consume.

Results: The paper examines implications of the asymmetric language positions of the groups for sectoral choices and within-group inequalities and effects of weights on the two types of education on levels and inter-group inequalities of skill, earnings, and consumption. Main results can be summarized as follows.

First, while the national and local sectors are indifferent to the majority, minority individuals with relatively large wealth choose the national sector and less wealthy ones choose the local sector. That is, the national sector is "ranked" higher than the local sector only for the minority. The reason is that, unlike the majority and unlike working in the local sector, education is a prerequisite for the minority to work in the national sector. Stemming from the contrasting sectoral choices of the groups, a change in within-group wealth inequality tends to have greater effects on within-group inequalities in earnings and consumption of the minority than the inequalities of the majority.

Second, regarding skill development of the minority, balanced allocation of expenditure to common language education and mother tongue education is crucial for those with limited wealth who choose the local sector: when the allocation is very biased, their return to educational investment becomes negative and they do not spend on education. ${ }^{2}$ As for future national sector workers, common-language-only education could maximize the skill for the sector under certain conditions. However, considering that mother tongue skill is important for off-the-job activities such as childrearing, the dual education is beneficial for them too. Thus, the result is mostly consistent with the aforementioned consensus among specialists on language and education.

Third, regarding earnings net of educational expenditure and consumption of the minority, balanced dual education is desirable only when the country has favorable conditions (i.e., sectoral productivities and the effectiveness of education are sufficiently high, and the proportion of those with adequate wealth for education is not very low) and only for those with sufficient wealth. Common-language-only education is always optimal for those with little wealth and, when the country's conditions are bad, it is optimal for all. ${ }^{3}$ In the real economy, the conditions are closely related to the level of economic and social development. Thus, the result implies that, in general,

\footnotetext{
${ }^{1}$ For analytical tractability, the model abstracts from non-language skill. Main results would not change by including non-language skill as an input of human capital production functions.

${ }^{2}$ This result might appear implausible, since most of students take some education even in poor countries. The difference from real economy arises because, for analytical tractability, the model abstracts from non-investment motives for attending school, including consumption motives (joys of studying or attending school) and social motives (pleasure of doing what friends do, pressure from family members or the community to attend school).

${ }^{3}$ It is also found that common-language-only education is definitely better than introducing mother tongue education on a small scale: the latter does not change human capital of those choosing the local sector, lowers human capital of those choosing the national sector, and lowers net earnings and consumption of all.
} 
if the level of development is low, common-language-only education is desirable in terms of the economic outcomes, otherwise, balanced bilingual education is desirable except for the very poor.

Finally, because the majority are not affected by weights on the two types of education the minority receive, the above results directly apply to inter-group inequalities in skill, net earnings, and consumption. For example, switching from exclusively common language education to balanced dual education is able to curtail the inter-group inequalities in earnings and consumption (except at the bottom of the distributions) only when the country has favorable conditions.

Policy implications: These results suggest that policies that bring good outcomes in both skill development and consumption to every minority individual and reduce the inter-group inequalities are different depending on conditions of the country.

When the conditions are favorable, which is likely to be the case when the level of development is sufficiently high, the government should implement balanced bilingual education together with redistributive policies that support educational investment of those with little wealth, such as income transfers and tuition subsidy. ${ }^{4}$ Without the latter policies, the very poor lose economically from the implementation of the dual education, because they cannot spend sufficiently enough on education to benefit from it.

By contrast, when the conditions are unfavorable, which is likely to be true when the level of development is low, what the government should conduct in the first place is policies changing the adverse conditions: improving the productivities and the effectiveness of education. If these policies are conducted on a sufficient scale, dual education coupled with redistribution towards the very poor can bring good outcomes to all. On the other hand, if the government cannot implement these policies on a sufficient scale for budgetary or other reasons, it faces a difficult choice between educational and economic outcomes.

Finally, the result that a change in within-group wealth inequality tends to have greater effects on earnings and consumption inequalities of the minority than the inequalities of the majority suggests that redistributive policies that increase access to education of the poor would be more important for the minorty than for the majority.

Note that the model does not take into account several important effects of the choice of language in education. Mother tongue education would raise ethnic language skill and contribute to the accumulation of social capital in the local ethnic community. It might also stimulate political participation and increase support for democracy (Albaugh, 2016). Common language education, on the other hand, would help people identify with the nation and contribute to national unity and stability. It might also reduce linguistic diversity and promote public goods provision and economic growth (Desmet, Ortuño-Ortín, and Wacziarg, 2012). Policy implementation in the actual society needs to take into account these effects as well.

Related literature: To the author's knowledge, this paper, along with Yuki (2018), is the first attempt to examine theoretically how weights on two types of education, common language education and mother tongue education, affect human capital, net earnings, and consumption of individuals with different family income. Several works examine related issues theoretically.

Pool (1991) examines the choice of official language(s) in a multilingual society in which earnings are exogenous, adopting a non-native official language is costly, and when there are multiple official languages, translation among the languages is costly and financed by tax. He shows that there exists efficient and fair choice of official language(s), if appropriate inter-group redistribution is conducted. Lazear (1999) constructs a model of a multilingual society, in which individuals heterogenous

\footnotetext{
${ }^{4}$ Of course, given weights on the two types of education, redistribution towards individuals without enough wealth for education would be desirable, as long as their return to education is positive. Rather, what is stated here is that redistribution towards the very poor is needed to implement the dual education, i.e., to choose balanced weights.
} 
in costs of learning non-native languages decide whether to master languages of other groups, each individual randomly matches with another individual and the match produces goods only when the pair can use the same language. He derives several implications of the model and empirically examines them. Ortega and Tangerås (2008) model a society of two language groups in which the politically dominant group determine the type(s) of schools accessible to each group (monolingual school in either language and bilingual school), individuals decide whether to attend school, and goods are produced from bilateral random matching as in Lazear (1999). They show that the dominant group choose laissez-faire or restrict access to schools using the language of the subordinate group, while the subordinate prefer schools using their own language.

Besides examining different issues, the present work is distinct from these works in the following respects. First, in the present work, individuals within each ethnic group are heterogenous in wealth available for education, while in the preceding works, they are either homogenous (Pool; Ortega and Tangerås) or heterogenous in costs of learning non-native languages, which would capture difference in innate ability (Lazear). This work adopts the different setting, because it mainly focuses on developing countries where family wealth is a critical determinant of educational investment even in basic education, whereas the existing works largely focus on developed countries. Second, individuals choose a sector that brings higher net earnings in this work, whereas, in the previous works, individuals randomly get a "job" (Lazear; Ortega and Tangerås) or earnings are exogenous (Pool). As in the present model, those who have limited (abundant) wealth to spend on education tend to choose the local (national) sector in the real economy. Having a more realistic job choice is important in the model, because the heterogenous effect of the education policy on earnings of individuals with different wealth depends on their job choices.

Main differences from Yuki (2018) are as follows. First, common language is the native language of the dominant group in the present work, whereas ethnic groups are symmetric and common language is not a native language of any group in Yuki (2018). Such setting is relevant to many subSaharan Africa countries where common language is the language of the former colonizer. Second, because of the setting of symmetric groups, Yuki (2018) does not examine effects of the education language policy on between-group inequalities. It does not explore implications of asymmetric language positions of the two groups for sectoral choices and within-group inequalities either. Third, human capital production functions of the present model exhibit decreasing returns to educational expenditure, which are standard and more plausible than linear functions with the upper bound on the expenditure of Yuki (2018).

In education and linguistics, many works study the effect of educational language policy on academic achievement of students, and a general consensus among researchers is that placing emphasis on ethnic language education at least in primary education is important for skill development (Ball, 2011; Heugh, 2011). In economics, a small number of works examine effects on educational and labor market outcomes empirically. Jain (2017) examines the effect on academic outcomes, using data of South India, where primary education is largely taught in the official language of the state. By comparing districts where the official language matched the district's language and ones where it did not, he finds that mismatched districts had lower literacy rates and college graduation rates, but after reorganization of states on linguistic lines, the previously mismatched districts caught up with other districts. Ramachandran (2017) finds that the reform in Ethiopia which introduced mother tongue instruction in primary education has positive effects on reading skill and years of schooling. These findings are consistent with the model's result on the educational outcome.

As for labor market outcomes, Angrist and Lavy (1997) find that the policy change of the 1980s in Morocco that replaced French with Arabic as the medium of instruction in post-primary education greatly lowered returns to schooling. Cappellari and Di Paolo (2018) analyze the effects 
of the 1983 bilingual education reform in Catalonia, which substantially increased the weight on Catalan in mandatory education (from a very low weight to a slightly higher weight than Spanish), and find a positive effect on earnings. Consistent with the model's result on earnings, these findings suggest that a large increase in the weight on local language education lowers wages in a developing country (Morocco) and raises wages in a developed region (Catalonia). Chakraborty and Bakshi (2016) find that the policy change in the Indian state of West Bengal that abolished English education in primary schools has a large negative effect on wages. This is consistent with the model's result that education very biased towards local language skill results in low earnings.

Organization of the paper: Section 2 presents the model. Section 3 examines the unconstrained case in which everyone has enough wealth for education, and Section 4 considers the general case in which educational investment of some individuals are wealth-constrained. Section 5 discusses policy implications of the results. Section 6 concludes. Appendix A explains how endogenous variables are determined in the general case, and Appendix B presents proofs of lemmas and propositions of the unconstrained case. Web Appendix $\mathrm{C}$ contains those of the general case.

\section{Model}

Consider a bilingual society populated by two ethnic groups, groups 1 and 2, and composed of three sectors, the national sector and group-specific local sectors. The local sector of each group produces group-specific final goods using intermediate goods produced by the national sector and the group's labor, while the national sector produces intermediate goods using labor of both groups. The alternative interpretation is that individuals consume two kinds of final goods, goods produced by the national sector and goods produced by the local sector of their group using labor only.

In the real economy, national sector jobs correspond to many jobs in companies doing business nationwide and jobs involving communications with other groups, which require common language skill, while local sector jobs correspond to many jobs in companies doing business locally and jobs involving communications with local customers, such as jobs in retail, food service, and personal care, which require local ethnic language skill. The interpretation of the local sectors as sectors producing group-specific final goods reflects the fact that these services are dominant in the final stage of the production process.

The production function of the local sector of group $i(i=1,2)$ is ${ }^{5}$

$$
Y_{i}=\left(T_{i} H_{i L}\right)^{\alpha}\left(Y_{i N}\right)^{1-\alpha}, \alpha \in(0,1),
$$

where $H_{i L}$ is the total human capital of workers in the sector, whose determination is explained later, $T_{i}$ is the sector's constant productivity, and $Y_{i N}$ is the amount of intermediate goods used. The production function implies that both the human capital and intermediate goods are essential but they are substitutable to some degree in the production of the final goods.

The production function of the national sector is

$$
Y_{N}=T_{N}\left(H_{1 N}+H_{2 N}\right)
$$

where $H_{i N}$ is the total human capital of group $i$ workers in the sector and $T_{N}$ is the sector's constant productivity. Workers of the two groups are perfectly substitutable in the production of the intermediate goods.

Markets are perfectly competitive. Let the intermediate good be the numeraire. Then, from (2), the wage rate per human capital of workers in the national sector is

\footnotetext{
${ }^{5}$ Under the alternative interpretation that individuals consume two kinds of final goods, this is the utility function and the production function of the local sector is $Y_{i}=T_{i} H_{i L}$.
} 


$$
w_{N}=T_{N}
$$

Denote the relative price of the final good of group $i$ by $P_{i}$ and the wage rate per human capital of local sector workers of group $i$ by $w_{i L}$. Since the profit of the final good producer is $P_{i} Y_{i}-w_{i L} H_{i L}-Y_{i N}$, from the first-order conditions of the profit maximization problem,

$$
\begin{aligned}
P_{i} \frac{\partial Y_{i}}{\partial H_{i L}} & =w_{i L} \Leftrightarrow P_{i} \frac{\alpha Y_{i}}{H_{i L}}=w_{i L}, \\
P_{i} \frac{\partial Y_{i}}{\partial Y_{i N}} & =1 \Leftrightarrow P_{i} \frac{(1-\alpha) Y_{i}}{Y_{i N}}=1 .
\end{aligned}
$$

From these equations,

$$
w_{i L}=\frac{\alpha}{1-\alpha} \frac{Y_{i N}}{H_{i L}} .
$$

Because the final goods are group-specific, there are no inter-group transactions of the goods. Thus, the demand for intermediate goods of a group must be equal to the amount of the goods produced by the group's workers: ${ }^{6}$

$$
Y_{i N}=T_{N} H_{i N} .
$$

By substiting the above equation into (1) and (6), the output of the final goods and the wage rate of local sector workers can be expressed as functions of $H_{i N}$ and $H_{i L}$ :

$$
\begin{aligned}
Y_{i} & =\left(T_{i} H_{i L}\right)^{\alpha}\left(T_{N} H_{i N}\right)^{1-\alpha}, \\
w_{i L} & =\frac{\alpha}{1-\alpha} \frac{T_{N} H_{i N}}{H_{i L}} .
\end{aligned}
$$

From (5), (7), and (8), the relative price of the final good is also expressed as a function of the human capital variables.

$$
\begin{aligned}
P_{i} & =\frac{1}{1-\alpha} \frac{Y_{i N}}{Y_{i}} \\
& =\frac{1}{1-\alpha}\left(\frac{T_{N} H_{i N}}{T_{i} H_{i L}}\right)^{\alpha} .
\end{aligned}
$$

The national sector requires the skill to use the common language, and the local sector of an ethnic group requires the skill to use its ethnic language. Group 1 is supposed to be in the large majority or has held a dominant position historically and thus the ethnic language of the group is the common language in the society. This implies that the skills required in the two sectors are the same for group 1, while they are different for group 2 .

Each person has wealth (endowment) $a$ to expend on education for developing these skills. Let the amount of educational spending be $e$. Group 1 individuals develop the skill to use their ethnic language, whereas group 2 individuals must build up both mother tongue skill and common language skill. It is assumed that group 2 individuals cannot choose the allocation of spending over the development of the two types of skills, which is fixed reflecting the fact that weights on common language education and local language education in basic education (primary and lower secondary education) are mostly determined by the government.

The human capital production function of group 1 individuals is

$$
h_{1} \equiv h_{1 N}=h_{1 L}=(\bar{l}+e)^{\gamma}, \gamma \in(0,1), \bar{l}>0
$$

\footnotetext{
${ }^{6}$ Note, however, that a part of intermediate goods used to produce the final goods of a group is produced by the other group. The proportion of intermediate goods used by a group that is produced by the own group is $\frac{H_{i N}}{H_{1 N}+H_{2 N}}$, which becomes smaller as $H_{i N}$ is smaller.
} 
where $h_{1 N}$ and $h_{1 L}$ are human capital in the national sector and in the local sector respectively, which are same for group 1 , and $\bar{l}$ is a constant. The level of human capital is positive without education, reflecting the fact that mother tongue skill is developed partly at home.

The human capital production functions of group 2 individuals are

$$
\begin{aligned}
h_{2 N} & =\left[\delta_{N}(1-s) e\right]^{\gamma}, s \in[0,1], \\
h_{2 L} & =\left(\bar{l}+\delta_{L} s e\right)^{\gamma},
\end{aligned}
$$

where $s \in[0,1]$ is the proportion of $e$ allocated to the development of the skill for the local sector, and $\delta_{N}\left(\delta_{L}\right)$ is the productivity of education technology for common language skill (mother tongue skill). ${ }^{7}$ The function for the local sector is similar to that of group 1, while the function for the national sector is different: the level of the skill for the sector is zero without education, because the common language is not their mother tongue. ${ }^{8} \delta_{N}<\delta_{L}$ would be reasonable considering higher cost effectiveness of mother tongue education in skill development (Vaillancourt and Grin, 2000).

A person with wealth $a$ can spend at most $e=a$ on education due to the absence of credit market to finance education. ${ }^{9}$ The next section analyzes the case in which no one is bound by the wealth constraint on educational investment, that is, everyone has enough wealth to make optimal spending. This case would not be relevant to many developing countries in which students must rely on limited family wealth to pay for study materials, commuting cost, uniforms, and supplementary education even when public schools do not charge tuitions. Hence, Section 4 examines the general case in which some people may not have enough wealth for optimal investment. Further, the section analyzes important issues not present in the unconstrained case.

After spending on education, each person chooses a sector to work and receives earnings, which, together with remaining wealth (endowment) $a-e$, are spent on final goods for consumption.

\section{Unconstrained case}

This section considers the case in which everyone has enough wealth to make optimal educational spending. Although this case may not be realistic to many developing countries, it is simpler to examine than the general case and helpful to understand the analysis of the case in the next section.

\subsection{Group 1}

First, we examine how group 1 variables are determined. Because group 1's human capital is the same in the national and local sectors, the wage rates per human capital of the sectors must be equal. Thus, from (3) and (9),

$$
w_{N}=w_{1 L}=T_{N}=\frac{\alpha}{1-\alpha} \frac{T_{N} H_{1 N}}{H_{1 L}} .
$$

Hence, the ratio of total human capital of the two sectors is constant:

\footnotetext{
${ }^{7}$ The productivity of education for group 1 is normalized to 1 because main results do not depend on it.

${ }^{8}$ For analytical tractability, the model abstracts from non-language skill. When non-language skill is also an input of human capital production functions, the functions should be such that language skill and non-language skill are complementary, i.e., language skill stimulates development of non-language skill and vice versa; and human capital is positive even without receiving education of non-language skill, i.e., knowledge and social skill are partly acquired outside school. A natural extension of the original production functions satisfying these properties is: $h_{1}=(\bar{l}+q e)^{\gamma}\left[\bar{l}_{n}+(1-q) e\right]^{\gamma_{n}}$ for group $1, h_{2 N}=\left[\delta_{N} q(1-s) e\right]^{\gamma}\left[\bar{l}_{n}+(1-q) e\right]^{\gamma_{n}}$ and $h_{2 L}=\left(\bar{l}+\delta_{L} q s e\right)^{\gamma}\left[\bar{l}_{n}+(1-q) e\right]^{\gamma_{n}}$ for group 2, where $q \in[0,1]$ is the fixed proportion of $e$ allocated to language education, $\gamma_{n} \in(0,1)$, and $\bar{l}_{n}>0$ is a constant. As explained in footnote 12 in Section 3, main results would not change under such specification.

${ }^{9}$ Introducing the government that partially finances education complicates the analysis but would not affect results qualitatively.
} 


$$
\frac{H_{1 N}}{H_{1 L}}=\frac{1-\alpha}{\alpha} .
$$

By substituting this equation into (10), the relative price of the final good of group 1 is

$$
P_{1}=\frac{1}{\alpha^{\alpha}(1-\alpha)^{1-\alpha}}\left(\frac{T_{N}}{T_{1}}\right)^{\alpha} .
$$

Because endowment (wealth) can alternatively be used for final good consumption, the income (net of the cost of education) maximization problem of a group 1 individual is

$$
\max _{e}\left\{w_{N} h_{1}-P_{1} e\right\}=\max _{e}\left\{T_{N}(\bar{l}+e)^{\gamma}-P_{1} e\right\} .
$$

From the first-order condition, ${ }^{10}$

$$
\gamma T_{N}(\bar{l}+e)^{\gamma-1}=P_{1} \Leftrightarrow \bar{l}+e=\left(\frac{\gamma T_{N}}{P_{1}}\right)^{\frac{1}{1-\gamma}} .
$$

Thus, the optimal educational spending, denoted $e_{1}^{*}$, equals

$$
e_{1}^{*}=\left[\gamma\left(\alpha T_{1}\right)^{\alpha}\left((1-\alpha) T_{N}\right)^{1-\alpha}\right]^{\frac{1}{1-\gamma}}-\bar{l} .
$$

By substituting (15) and (17) into (16), earnings net of the cost of education equal

$$
\begin{aligned}
& T_{N}\left[\gamma\left(\alpha T_{1}\right)^{\alpha}\left((1-\alpha) T_{N}\right)^{1-\alpha}\right]^{\frac{\gamma}{1-\gamma}}-\frac{1}{\alpha^{\alpha}(1-\alpha)^{1-\alpha}}\left(\frac{T_{N}}{T_{1}}\right)^{\alpha}\left\{\left[\gamma\left(\alpha T_{1}\right)^{\alpha}\left((1-\alpha) T_{N}\right)^{1-\alpha}\right]^{\frac{1}{1-\gamma}}-\bar{l}\right\} \\
& =\frac{1}{\alpha^{\alpha}(1-\alpha)^{1-\alpha}}\left(\frac{T_{N}}{T_{1}}\right)^{\alpha}\left\{(1-\gamma)\left[\gamma^{\gamma}\left(\alpha T_{1}\right)^{\alpha}\left((1-\alpha) T_{N}\right)^{1-\alpha}\right]^{\frac{1}{1-\gamma}}+\bar{l}\right\} .
\end{aligned}
$$

The consumption of a group 1 worker with wealth (endowment) $a$ equals, from (15) and (18),

$$
\begin{aligned}
c_{1}^{*}(a) & =\frac{w_{N} h_{1}^{*}-P_{1} e_{1}^{*}}{P_{1}}+a \\
& =(1-\gamma)\left[\gamma^{\gamma}\left(\alpha T_{1}\right)^{\alpha}\left((1-\alpha) T_{N}\right)^{1-\alpha}\right]^{\frac{1}{1-\gamma}}+\bar{l}+a .
\end{aligned}
$$

\subsection{Group 2}

\subsubsection{Individuals choosing the national sector}

Now, the determinations of group 2 variables are analyzed. First, consider those who become national sector workers. Their income maximization problem is

$$
\max _{e}\left\{w_{N} h_{2 N}-P_{2} e\right\}=\max _{e}\left\{T_{N}\left[\delta_{N}(1-s) e\right]^{\gamma}-P_{2} e\right\} .
$$

The first-order condition is

$$
\gamma T_{N} \frac{\left[\delta_{N}(1-s) e\right]^{\gamma}}{e}-P_{2}=0 .
$$

From the above equation, their educational spending equals

$$
\begin{aligned}
e_{2 N}^{*} & =\left\{\frac{\gamma T_{N}\left[\delta_{N}(1-s)\right]^{\gamma}}{P_{2}}\right\}^{\frac{1}{1-\gamma}} \\
& =\left\{(1-\alpha) T_{2}^{\alpha} T_{N}{ }^{1-\alpha} \gamma\left[\delta_{N}(1-s)\right]^{\gamma}\left(\frac{H_{2 N}}{H_{2 L}}\right)^{-\alpha}\right\}^{\frac{1}{1-\gamma}}(\text { from (10)). }
\end{aligned}
$$

\footnotetext{
${ }^{10}$ For $e>0$ to be optimal, it is assumed that $T_{N}$ and $T_{1}$ are large enough that $\gamma T_{N}(\bar{l})^{\gamma-1}-P_{1}>0 \Leftrightarrow \gamma\left(\alpha T_{1}\right)^{\alpha}((1-$ $\left.\alpha) T_{N}\right)^{1-\alpha}(\bar{l})^{\gamma-1}>1$ holds.
} 
Since their human capital is zero without education, $e_{2 N}^{*}>0$ holds, unless $s=1$.

By substituting (10) and (21) into (20), their earnings net of the cost of education equal

$$
w_{N} h_{2 N}^{*}-P_{2} e_{2 N}^{*}=(1-\gamma)\left\{T_{N}\left[(1-\alpha) \gamma \delta_{N}(1-s)\left(\frac{T_{N} H_{2 N}}{T_{2} H_{2 L}}\right)^{-\alpha}\right]^{\gamma}\right\}^{\frac{1}{1-\gamma}} .
$$

\subsubsection{Individuals choosing the local sector}

Next, consider those who become local sector workers. Their income maximization problem is

$$
\max _{e}\left\{w_{2 L} h_{2 L}-P_{2} e\right\}=\max _{e}\left\{w_{2 L}\left(\bar{l}+\delta_{L} s e\right)^{\gamma}-P_{2} e\right\} .
$$

From the first-order condition, when $\gamma \delta_{L} s w_{2 L}(\bar{l})^{\gamma-1}-P_{2}>0$, i.e., when positive $e$ is optimal,

$$
\gamma \delta_{L} s w_{2 L}\left(\bar{l}+\delta_{L} s e\right)^{\gamma-1}=P_{2} \Leftrightarrow \bar{l}+\delta_{L} s e=\left(\frac{\gamma \delta_{L} s w_{2 L}}{P_{2}}\right)^{\frac{1}{1-\gamma}} .
$$

Thus, their educational spending equals

$$
\begin{aligned}
e_{2 L}^{*} & =\frac{1}{\delta_{L} s}\left[\left(\frac{\gamma \delta_{L} s w_{2 L}}{P_{2}}\right)^{\frac{1}{1-\gamma}}-\bar{l}\right] \\
& =\frac{1}{\delta_{L} s}\left[\left(\frac{\gamma \delta_{L} s \alpha Y_{2}}{H_{2 L}}\right)^{\frac{1}{1-\gamma}}-\bar{l}\right](\text { from }(5)) \\
& =\frac{1}{\delta_{L} s}\left\{\left[\alpha \gamma \delta_{L} s T_{2}^{\alpha} T_{N}{ }^{1-\alpha}\left(\frac{H_{2 N}}{H_{2 L}}\right)^{1-\alpha}\right]^{\frac{1}{1-\gamma}}-\bar{l}\right\} .
\end{aligned}
$$

By substituting (4), (10), and (24) into (23), their earnings net of the cost of education equal

$$
\begin{aligned}
w_{2 L} h_{2 L}^{*}-P_{2} e_{2 L}^{*} & =P_{2}\left[\frac{\alpha Y_{2}}{H_{2 L}}\left(\bar{l}+\delta_{L} s e_{2 L}^{*}\right)^{\gamma}-e_{2 L}^{*}\right] \\
& =\frac{1}{1-\alpha}\left(\frac{T_{N} H_{2 N}}{T_{2} H_{2 L}}\right)^{\alpha}\left\{(1-\gamma)\left[\left(\gamma \delta_{L} s\right)^{\gamma} \alpha T_{2}^{\alpha} T_{N}{ }^{1-\alpha}\left(\frac{H_{2 N}}{H_{2 L}}\right)^{1-\alpha}\right]^{\frac{1}{1-\gamma}}+\frac{\bar{l}}{\delta_{L} s}\right\}
\end{aligned}
$$

By contrast, when $\gamma \delta_{L} s w_{2 L}(\bar{l})^{\gamma-1}-P_{2} \leq 0$, not taking education is optimal, i.e., $e_{2 L}^{*}=0$. In this case, from (9), their earnings equal

$$
w_{2 L}(\bar{l})^{\gamma}=\frac{\alpha}{1-\alpha} T_{N} \frac{H_{2 N}}{H_{2 L}}(\bar{l})^{\gamma} .
$$

\subsubsection{Indifference condition}

Since everyone has enough wealth to take optimal education for either sector, individuals are indifferent between the sectors, which implies that net earnings of the two sectors are equal.

Thus, when $e_{2 L}^{*}=0$, from (22) and (26), the following must hold:

$$
\begin{aligned}
& (1-\gamma)\left\{T_{N}\left[(1-\alpha) \gamma \delta_{N}(1-s)\left(\frac{T_{N} H_{2 N}}{T_{2} H_{2 L}}\right)^{-\alpha}\right]^{\gamma}\right\}^{\frac{1}{1-\gamma}}=\frac{\alpha}{1-\alpha} T_{N} \frac{H_{2 N}}{H_{2 L}}(\bar{l})^{\gamma} \\
& \Leftrightarrow \frac{H_{2 N}}{H_{2 L}}=\left\{\left(\frac{1-\alpha}{\alpha} \frac{1-\gamma}{(\bar{l})^{\gamma}}\right)^{1-\gamma}\left[(1-\alpha) \gamma \delta_{N}(1-s) T_{2}^{\alpha} T_{N}{ }^{1-\alpha}\right]^{\gamma}\right\}^{\frac{1}{1-\gamma(1-\alpha)}} .
\end{aligned}
$$


When $e_{2 L}^{*}>0$, from (22) and (25), the indifference condition is

$$
(1-\gamma)\left\{T_{N}\left[(1-\alpha) \gamma \delta_{N}(1-s)\left(\frac{T_{N} H_{2 N}}{T_{2} H_{2 L}}\right)^{-\alpha}\right]^{\gamma}\right\}^{\frac{1}{1-\gamma}}=\frac{\left(\frac{T_{N} H_{2 N}}{T_{2} H_{2 L}}\right)^{\alpha}}{1-\alpha}\left\{(1-\gamma)\left[\left(\gamma \delta_{L} s\right)^{\gamma} \alpha T_{2}^{\alpha} T_{N}{ }^{1-\alpha}\left(\frac{H_{2 N}}{H_{2 L}}\right)^{1-\alpha}\right]^{\frac{1}{1-\gamma}}+\frac{\bar{l}}{\delta_{L} s}\right\}
$$

Once $\frac{H_{2 N}}{H_{2 L}}$ is determined from the indifference condition, $e_{2 N}^{*}, e_{2 L}^{*}$ when positive, and $P_{2}$ are determined from $(21),(24)$, and (10), respectively. ${ }^{11}$

Finally, consumption of a group 2 individual with wealth (endowment) $a$ is determined from

$$
\begin{aligned}
c_{2}^{*}(a) & =\frac{w_{N} h_{2 N}^{*}-P_{2} e_{2 N}^{*}}{P_{2}}+a \\
& =(1-\alpha)\left(\frac{T_{N} H_{2 N}}{T_{2} H_{2 L}}\right)^{-\alpha}(1-\gamma)\left\{T_{N}\left[(1-\alpha) \gamma \delta_{N}(1-s)\left(\frac{T_{N} H_{2 N}}{T_{2} H_{2 L}}\right)^{-\alpha}\right]^{\gamma}\right\}^{\frac{1}{1-\gamma}}+a \quad \text { (from (10) and } \\
& =(1-\gamma)\left\{(1-\alpha)\left[\gamma \delta_{N}(1-s)\right]^{\gamma} T_{2}{ }^{\alpha} T_{N}{ }^{1-\alpha}\left(\frac{H_{2 N}}{H_{2 L}}\right)^{-\alpha}\right\}^{\frac{1}{1-\gamma}}+a .
\end{aligned}
$$

\subsection{Results}

Based on the above analysis, this section examines the following questions: for group 2 individuals, what is a desirable combination of the two types of education in terms of future earnings and consumption, and what is a desirable combination in terms of skill development? Because skill, earnings, and consumption of group 1 individuals are not affected by the education policy of group 2 , they are mentioned only in relation to those of group 2 individuals.

The previous subsection shows that educational spending of those who choose the local sector subsequently is either zero or positive. The next lemma shows whether $e_{2 L}^{*}=0$ or $e_{2 L}^{*}>0$ depends on the weight on local language education, $s$.

Lemma 1 Suppose that all group 2 individuals have enough wealth (endowment) for education and $T_{2}$ and $T_{N}$ are not extremely low. Then,

(i) There exist two critical values of $s$, denoted $\underline{s}, \bar{s} \in(0,1)(\underline{s}<1-\gamma(1-\alpha)<\bar{s})$, such that those who choose the local sector do not spend on education, i.e., $e_{2 L}^{*}=0$, for $s \leq \underline{s}$ and $s \geq \bar{s}$, while, for $s \in(\underline{s}, \bar{s})$, their educational spending is positive, i.e., $e_{2 L}^{*}>0$.

(ii) $\underline{s}(\bar{s})$ decreases (increases) with $T_{2}, T_{N}, \delta_{N}$, and $\delta_{L}$.

Figure 1 illustrates the result. When the proportion of educational spending allocated to education useful in the local sector is very low or very high, i.e., for $s \leq \underline{s}$ and $s \geq \bar{s}$, those who choose the local sector subsequently do not spend on education, i.e., $e_{2 L}^{*}=0$, while when the allocation is relatively balanced, i.e., for $s \in(\underline{s}, \bar{s})$, their spending is positive, i.e., $e_{2 L}^{*}>0 .{ }^{12}$

\footnotetext{
${ }^{11}$ Further, once $\frac{H_{2 N}}{H_{2 L}}$ is determined, $H_{2 N}, H_{2 L}$, and the number of workers in each sector, $L_{2 N}$ and $L_{2 L}=L_{2}-L_{2 N}$, are determined from $H_{2 N}=\left[\delta_{N}(1-s) e_{2 N}^{*}\right]^{\gamma} L_{2 N}$ and $H_{2 L}=\left(\bar{l}+\delta_{L} s e_{2 L}^{*}\right)^{\gamma} L_{2 L}$. In particular, when $e_{2 L}^{*}=0$, $\frac{L_{2 N}}{L_{2 L}}=\frac{1-\alpha}{\alpha}(1-\gamma)$, while when $e_{2 L}^{*}>0, \frac{L_{2 N}}{L_{2 L}}=\left[\frac{H_{2 N}}{H_{2 L}}\left(\frac{\alpha}{1-\alpha} \frac{\delta_{L} s}{\delta_{N}(1-s)}\right)^{\gamma}\right]^{\frac{1}{1-\gamma}}$.

${ }^{12}$ When human capital production functions include non-language skill as an input and are given by the equations in footnote 8 of Section 2, different from the original specification, when $T_{N}, T_{2}, \delta_{N}$, and $\delta_{L}$ are high, $e_{2 L}^{*}>0$ holds at $s=0$, i.e., $\underline{s}$ does not exist. This is because the return to educational expenditure on non-language skill does not depend on $s$ and is positive even at $e=0$. However, intuitive explanations of the lemmas (except the result on $\underline{s}$ ) and the propositions below remain unchanged, hence, main results would not change qualitatively.
} 


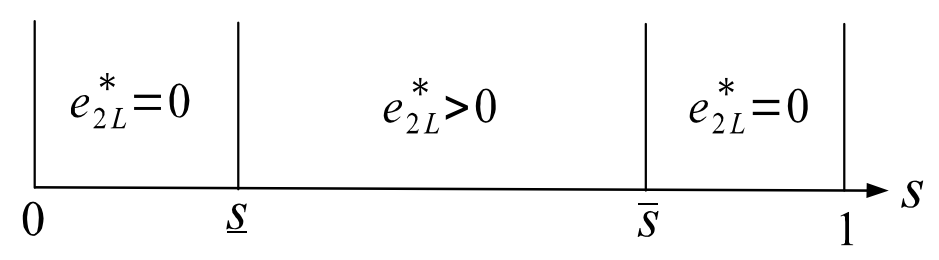

Figure 1: Lemma 1 (i)

The result can be understood from the marginal return to educational investment at $e=0$ (in unit of the final good) for those who choose the local sector, which equals, from (23), (8), and (10), $\left.\frac{w_{2 L}}{P_{2}} \frac{\partial h_{2 L}}{\partial e}\right|_{e=0}-1=\alpha\left(T_{2}\right)^{\alpha}\left(T_{N}\right)^{1-\alpha}\left(\frac{H_{2 N}}{H_{2 L}}\right)^{1-\alpha} \gamma \delta_{L} s(\bar{l})^{\gamma-1}-1 .{ }^{13}$ When $s$ is very low, the marginal return is negative, because the marginal effect of educational spending on human capital for the local sector $\left(\gamma \delta_{L} s(\bar{l})^{\gamma-1}\right)$ is very small, which dominates the sector's high wage rate due to large $\frac{H_{2 N}}{H_{2 L}} \cdot{ }^{14}$ By contrast, when $s$ is very high, the marginal return is negative, because $\frac{w_{2 L}}{P_{2}}$ becomes very low due to small $\frac{H_{2 N}}{H_{2 L}}$, which dominates a large marginal effect of $e$ on $h_{2 L}$. Higher productivities $T_{2}, T_{N}$ and higher effectiveness of education $\delta_{N}, \delta_{L}$ widen the range of $s$ over which educational investment is rewarding, because the wage rate is higher. ${ }^{15}$

The result that future local sector workers do not spend on education when $s$ is very low or very high might appear implausible, since the great majority of students take some education even in poor countries. The difference from the real economy arises because, for tractability, the model abstracts from motives for attending school other than the investment motive, including consumption motives (joys of studying or attending school) and social motives (pleasure of doing what friends do, pressure from family members or the community to attend school). The result, however, sheds light on an important source of poor academic performance of students in many countries. According to the result, students going to the local sector have weak incentive to study and thus perform badly, either because what they learn is mostly irrelevant to their future jobs in the local sector (when $s$ is very low) or because their future earnings are low due to deficient skill of workers in the complementary national sector (when $s$ is very high).

Next, the effect of $s$ on net earnings (in unit of the final good) and consumption of group 2 individuals is examined. The previous subsection shows that these variables of national sector workers depend negatively on $s$ and $\frac{H_{2 N}}{H_{2 L}}$ (equation (30)). How does $\frac{H_{2 N}}{H_{2 L}}$ depend on $s$ ?

Lemma 2 Suppose that all group 2 individuals have enough wealth for optimal education. Then, $\frac{H_{2 N}}{H_{2 L}}$ decreases with $s$.

Given $\frac{H_{2 N}}{H_{2 L}}$, an increase in $s$ makes the local sector more profitable than the national sector: an increase in $s$ lowers $h_{2 N}$ and thus net earnings in the national sector, while it raises $h_{2 L}$ and net earnings in the local sector when $e_{2 L}^{*}>0$ and do not affect these variables when $e_{2 L}^{*}=0$. By contrast, given $s$, a decrease in $\frac{H_{2 N}}{H_{2 L}}$ makes the national sector more attractive: a decrease in $\frac{H_{2 N}}{H_{2 L}}$ lowers $P_{2}$ (from a decrease in $\frac{Y_{2 N}}{Y_{2}}$ ) and raises net earnings in the national sector, $\frac{w_{N}}{P_{2}} h_{2 N}-e=$

\footnotetext{
${ }^{13}$ Thereafter, the marginal return to educational investment and net earnings are measured in unit of the final good, not in unit of the numeraire, the intermediate good.

${ }^{14}$ This is because small $s$ leads to large $h_{2 N}$ and small $h_{2 L}$. The next lemma, Lemma 2, formally shows that when $s$ is small, $\frac{H_{2 N}}{H_{2 L}}$ is large.

${ }^{15}$ Increases in $T_{N}$ and $\delta_{N}$ raise $\frac{w_{2 L}}{P_{2}}$, because workers in the local sector and the intermediate good produced in the national sector are complementary in the production of the final good.
} 
$\frac{T_{N}}{P_{2}}\left(\delta_{N}(1-s) e_{2 N}^{*}\right)^{\gamma}-e_{2 N}^{*}$, while it lowers $\frac{w_{2 L}}{P_{2}}$ and net earnings in the local sector. Hence, when $s$ increases, $\frac{H_{2 N}}{H_{2 L}}$ must decrease for workers to remain indifferent between the sectors.

Thus, the direct effect of $s$ on net earnings and consumption of national sector workers is negative, while the indirect effect through $\frac{H_{2 N}}{H_{2 L}}$ is positive. Which effect dominates? The following lemma examines the total effect when $e_{2 L}^{*}=0$ and when $e_{2 L}^{*}>0$ separately.

Lemma 3 Suppose that all group 2 individuals have enough wealth for education.

(i) When $e_{2 L}^{*}=0$, earnings net of the cost of education and consumption of group 2 individuals decrease with $s$.

(ii) When $e_{2 L}^{*}>0$, if $T_{N}, T_{2}, \delta_{N}$, and $\delta_{L}$ are low, net earnings and consumption of group 2 individuals decrease with $s$; otherwise, they decrease with $s$ for small $s$, increase with $s$ for intermediate $s$, and decrease with s for large $s$.

When future local sector workers do not spend on education, i.e., $e_{2 L}^{*}=0$, net earnings and consumption decrease as a greater proportion of educational expenditure is allocated to the development of the skill for the local sector. Earnings in the local sector decrease because $\frac{w_{2 L}}{P_{2}}$ falls due to lowered $\frac{H_{2 N}}{H_{2 L}}$ and $h_{2 L}$ remains unchanged. Since individuals are indifferent between the sectors, the same is true for net earnings in the national sector and consumption.

By contrast, when $e_{2 L}^{*}>0$, if the sectoral productivities $T_{2}, T_{N}$ and the effectiveness of education $\delta_{N}, \delta_{L}$ are low, net earnings and consumption decrease with the weight on local language education; otherwise, the effects of the weight on these variables are non-monotonic: they decrease with $s$ for small $s$, increase with $s$ for intermediate $s$, and decrease with $s$ again for large $s$. An increase in $s$ has a negative effect on net earnings in the national sector, $\frac{w_{N}}{P_{2}} h_{2 N}-e=\frac{T_{N}}{P_{2}}\left(\delta_{N}(1-s) e_{2 N}^{*}\right)^{\gamma}-e_{2 N}^{*}$, and consumption through a decrease in $h_{2 N}$, while it has a positive effect through decreases in $\frac{H_{2 N}}{H_{2 L}}$ and thus $P_{2}$. If $T_{N}, T_{2}, \delta_{N}$, and $\delta_{L}$ are low or if $s$ is small or large, the former effect dominates the latter effect and net earnings and consumption decrease with $s$; otherwise, the latter effect dominates and they increase with $s$.

The lemma implicitly assumes that either $e_{2 L}^{*}=0$ holds for any $s$ or $e_{2 L}^{*}>0$ holds for any $s$, which is not true as shown in Lemma 1 (Figure 1). By taking into account how $s$ affects whether $e_{2 L}^{*}=0$ or $e_{2 L}^{*}>0$, the next proposition examines the effect of $s$ on net earnings and consumption.

Proposition 1 Suppose that all group 2 individuals have enough wealth for education.

(i) If $T_{N}, T_{2}, \delta_{N}$, and $\delta_{L}$ are low, net earnings and consumption of group 2 individuals decrease with $s$ for any $s$ and thus they are maximized at $s=0$.

(ii) Otherwise, the net earnings and consumption decrease with $s$ for small $s$ and large $s,{ }^{16}$ and increase with $s$ for intermediate $s$. If $T_{N}, T_{2}, \delta_{N}$, and $\delta_{L}$ are sufficiently high, they are maximized at intermediate $s$, where $s$ maximizing these variables increases with $T_{N}, T_{2}, \delta_{N}$, and $\delta_{L} ;$ otherwise, they are maximized at $s=0$.

If the sectoral productivities $T_{2}, T_{N}$ and the productivities of education $\delta_{N}, \delta_{L}$ are low, net earnings and consumption of group 2 individuals always decrease with the weight on local language education and thus they are maximized when educational expenditure is exclusively allocated to common language education.

Otherwise, their net earnings and consumption decrease with $s$ for small $s$ and large $s$ and increase with $s$ for intermediate $s$. If $T_{2}, T_{N}, \delta_{N}$, and $\delta_{L}$ are sufficiently high, these variables are maximized at intermediate $s$, i.e., a balanced allocation of expenditure to mother tongue education

\footnotetext{
${ }^{16}$ They decrease with $s$ at least for $s \leq \underline{s}$ and $s \geq \min \{\alpha, \bar{s}\}$.
} 


\section{Net earnings}

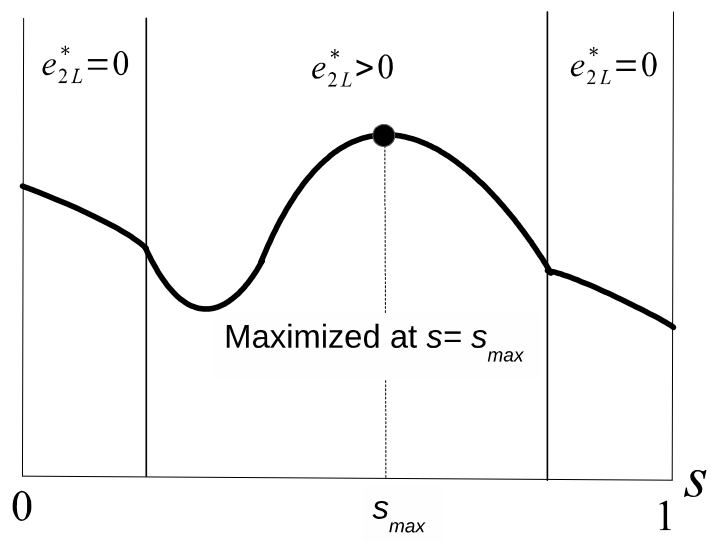

(a) Relatively high $T_{N}, T_{2}, \delta_{N}$, and $\delta_{L}$

\section{Net earnings}

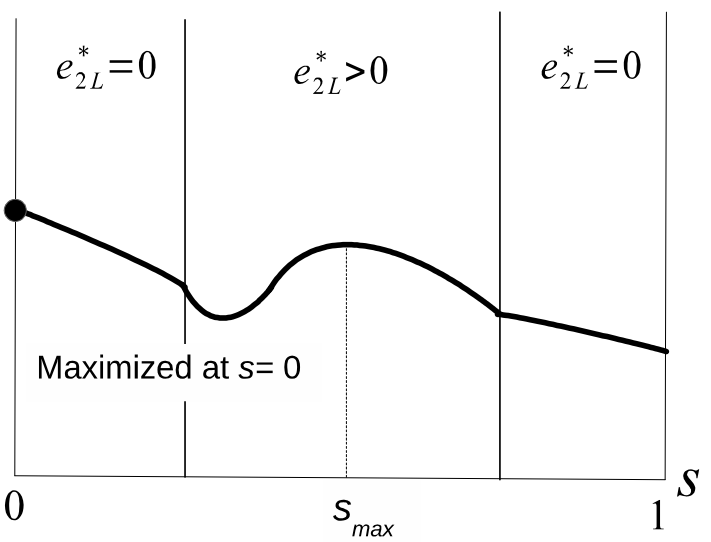

(b) Relatively low $T_{N}, T_{2}, \delta_{N}$, and $\delta_{L}$

Figure 2: Relationship between $s$ and net earnings (in unit of the final good) when $T_{N}, T_{2}, \delta_{N}$, and $\delta_{L}$ are not low of the unconstrained case (Proposition 1 (ii))

and common language education is economically desirable; otherwise, they are maximized at $s=0$. In other words, in order for bilingual education to be economically beneficial to the minority or subordinate group, the productivities and the effectiveness of education must be sufficiently high. Further, $s$ maximizing net earnings and consumption increases with the exogenous variables, i.e., as the sectoral productivities and the efficiencies of education become higher, the greater emphasis on mother tongue education becomes desirable. Figure 2 illustrates the relationship between $s$ and net earnings (in unit of the final good) for this case, (a) when $T_{2}, T_{N}, \delta_{N}$, and $\delta_{L}$ are relatively high and (b) when they are relatively low. (Similar figures can be drawn for consumption, which equals net earnings plus wealth.) In both cases, the shape of the graph is similar and net earnings are maximized at $s=0$ when $e_{2 L}^{*}=0$ and at $s=s_{\max }$ when $e_{2 L}^{*}>0$. However, in (a), the value at $s=s_{\max }$ is higher than the one at $s=0$, while the value at $s=0$ is higher in (b).

In the real economy, the sectoral productivities and the effectiveness of education of a country are closely related to the level of economic and social development. Hence, the result implies that, in general, if the level of development is low, net earnings and consumption are highest under common-language-only education, otherwise, they are highest under bilingual education. ${ }^{17}$ Angrist and Lavy (1997) find that the policy change of the 1980s in Morocco that replaced French with Arabic as the medium of instruction in post-primary education greatly lowered returns to schooling. ${ }^{18}$ Cappellari and Di Paolo (2018) analyze the effects of the 1983 education reform in Catalonia, which substantially increased the weight on Catalan in mandatory education (from a very low weight to a slightly higher weight than Spanish), and find a positive effect on earnings.

\footnotetext{
${ }^{17}$ When the model is applied to economies with different levels of development, it must be consistent with the fact that a large portion of educational expenditure is used for hiring teachers and thus the unit cost of education increases with the wage. This can be taken into account by replacing $e$ in human capital production functions with $e$ deflated by the unit cost of education, which increases with $w_{N}=T_{N}$. As far as the unit cost changes less proportionately to the wage, which is plausible since non-labor cost is non-negligible, qualitative results remain the same.

${ }^{18}$ Unlike the model, French is not the ethnic language of any group. However, at that time, French was the dominant language in many parts of the modern sector, such as public administration, foreign trade, and science and technology, and children of workers in these areas had a great advantage in acquiring French skills. Hence, the model's result would apply to this environment, if one interprets these children as the dominant group of the model.
} 
Consistent with the result, these findings suggest that a large increase in the weight on local language education lowers wages in a developing country (Morocco) and raises wages in a developed region (Catalonia).

Even among developing countries, the productivity of primary education is very different and explains most of the gap in cross-country test scores (Singh, 2020). ${ }^{19}$ The theoretical result implies that if a country has ineffective education system, net earnings and consumption are maximized under common-language-only education, otherwise, they are maximized under bilingual education.

What is crucial for the result is the assumption that human capital for the local sector is positive without education, i.e., $\bar{l}>0$. If $\bar{l}=0$, irrespective of values of $T_{2}, T_{N} \delta_{N}$, and $\delta_{L}, e_{2 L}^{*}>0$ holds for any positive $s$, net earnings and consumption are highest at intermediate $s=\alpha$, and they increase (decrease) with $s$ for smaller (greater) $s$. The assumption makes education unprofitable for future local sector workers when $s$ is very low or very high and causes net earnings and consumption to decrease with $s$ for low $s$, which could lead to these variables being maximized at $s=0$.

As mentioned in Introduction, what concerns experts on language and education is how weights on the two types of education affect skill development of students. Further, empirical evidence suggests that given income, human capital has positive effects on child-rearing, health, and among others. Hence, the next proposition examines the effect of $s$ on human capital for the national sector and the one for the local sector.

Proposition 2 Suppose that all group 2 individuals have enough wealth for education.

(i) $h_{2 L}^{*}$ is lowest when $s \leq \underline{s}$ and $s \geq \bar{s}$, while it is highest at $s=1-(1-\alpha) \gamma \in(\underline{s}, \bar{s})$ and increases (decreases) with $s$ for smaller (greater) $s \in(\underline{s}, \bar{s})$.

(ii) (a) If $T_{N}, T_{2}, \delta_{N}$, and $\delta_{L}$ are low, $h_{2 N}^{*}$ decreases with $s$ for any $s$ and is maximized at $s=0$.

(b) Otherwise, $h_{2 N}^{*}$ decreases with $s$ for small $s$ and large $s$ and increases with $s$ for intermediate $s .{ }^{20}$ If $T_{N}, T_{2}, \delta_{N}$, and $\delta_{L}$ are sufficiently high, $h_{2 N}^{*}$ is maximized at intermediate $s \in(\underline{s}, \alpha \gamma)$, where $s$ maximizing $h_{2 N}^{*}$ increases with $T_{N}, T_{2}, \delta_{N}$, and $\delta_{L}$; otherwise it is maximized at $s=0$.

Human capital for the local sector is lowest when $s$ is small or large enough that $e_{2 L}^{*}=0$, i.e., $s \leq \underline{s}$ and $s \geq \bar{s}$, and is highest when $s$ is at an intermediate level, i.e., $s=1-(1-\alpha) \gamma \in(\underline{s}, \bar{s})$. Figure 3 illustrates the relationship between $s$ and $h_{2 L}^{*}$.

In contrast, human capital for the national sector is highest when $s$ is zero or when $s$ is intermediate, i.e., $s \in(\underline{s}, \alpha \gamma)$. If $T_{N}, T_{2}, \delta_{N}$, and $\delta_{L}$ are low enough, $h_{2 N}^{*}$ is highest at $s=0$; otherwise it is highest at an intermediate $s$, where $s$ maximizing $h_{2 N}^{*}$ increases with $T_{N}, T_{2}, \delta_{N}$, and $\delta_{L}$, i.e., as the productivities and the effectiveness of education are higher, the greater emphasis on mother tongue education becomes beneficial for development of skill for the national sector. The relationship between $s$ and $h_{2 N}^{*}$ is similar to the one between $s$ and net earnings or consumption.

The result implies that balanced allocation of expenditure to common language education and mother tongue education is crucial for skill development of students who choose the local sector subsequently. By contrast, common-language-only education maximizes human capital for the national sector when the productivities and the effectiveness of education are low. However, for most people working in the national sector, the mother tongue is a major language at home and thus is important for child-rearing, health, and so forth. That is, the dual education is beneficial for them as well. Hence, the result is mostly consistent with a general consensus among specialists

\footnotetext{
${ }^{19}$ Based on panel data of four developing countries with widely differing test scores of primary school students (Ethiopia, India, Peru, and Vietnam), Singh (2020) shows that most of the differences in the student achievement are explained by cross-country differences in the productivity of primary education.

${ }^{20}$ It decreases with $s$ at least for $s \leq \underline{s}$ and $s \geq \min \{\alpha \gamma, \bar{s}\}$, where $\alpha \gamma<1-(1-\alpha) \gamma\left(s\right.$ maximizing $\left.h_{2 L}^{*}\right)$.
} 


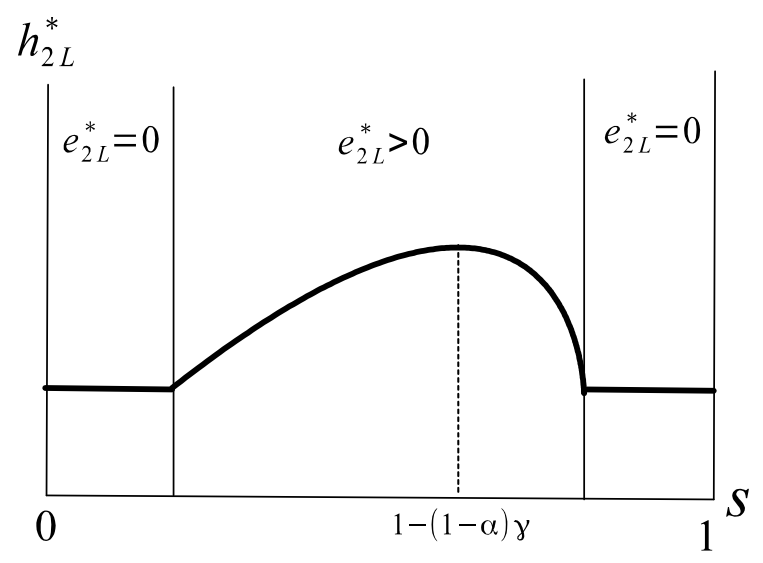

Figure 3: Relationship between $s$ and $h_{2 L}^{*}$ in the unconstrained case

on language and education that placing emphasis on ethnic language education at least in primary education is important for skill development (Ball, 2011; Heugh, 2011). It is also consistent with findings in economics (Jain, 2017; Ramachandran, 2017) that the significant introduction of mother tongue education increases academic skill and years of education.

The result that $h_{2 L}^{*}=\left(\bar{l}+\delta_{L} s e_{2 L}^{*}\right)^{\gamma}$ increases (decreases) with $s$ for small (large) $s \in(\underline{s}, \bar{s})$ can be explained as follows. An increase in $s$ has a direct positive effect on the human capital and indirect effects through a change in $e_{2 L}^{*}$. Because the marginal return to educational spending at $e=e_{2 L}^{*}$ is $\frac{w_{2 L}}{P_{2}} \frac{\partial h_{2 L}^{*}}{\partial e}-1=\alpha\left(T_{2}\right)^{\alpha}\left(T_{N}\right)^{1-\alpha}\left(\frac{H_{2 N}}{H_{2 L}}\right)^{1-\alpha} \gamma \delta_{L} s\left(\bar{l}+\delta_{L} s e_{2 L}^{*}\right)^{\gamma-1}-1=0$, an increase in $s$ affects $e_{2 L}^{*}$ positively through the increased marginal effect of $e$ on $h_{2 L}$, while it affects $e_{2 L}^{*}$ negatively through decreases in $\frac{H_{2 N}}{H_{2 L}}$ and thus the real wage rate. When $s$ is small (large), the positive effects dominate (are dominated by) the last negative effect and thus $h_{2 L}^{*}$ increases (decreases) with $s$.

Human capital for the national sector, $h_{2 N}^{*}=\left[(1-s) \delta_{N} e_{2 N}^{*}\right]^{\gamma}$, is also affected by $s$ through these effects, but signs of the effects are opposite from $h_{2 L}^{*}$ : the direct effect of $s$ on $h_{2 N}^{*}$ is negative; as for indirect effects through $e_{2 N}^{*}$, the effect through a change in the marginal effect of $e$ on $h_{2 N}$ is negative and the effect through changes in $\frac{H_{2 N}}{H_{2 L}}$ and thus $\frac{w_{2 N}}{P_{2}}$ is positive. If $T_{N}, T_{2}, \delta_{N}$, and $\delta_{L}$ are not low and $s$ is intermediate, the last positive effect dominates the negative effects and $h_{2 N}^{*}$ increases with $s$; otherwise, the negative effects dominate and $h_{2 N}^{*}$ decreases with $s$.

Finally, because group 1 individuals are not affected by weights on the two types of education group 2 students receive, the above results directly apply to inter-group inequalities in skill, net earnings, and consumption, which is summarized as the following corollary. In order to assure that group 1 individuals have higher net earning and consumption for given wealth, $T_{1} \geq T_{2}$ is assumed.

Corollary 1 Suppose that $T_{1} \geq T_{2}$ and all individuals have enough wealth for education.

(i) If $T_{N}, T_{2}, \delta_{N}$, and $\delta_{L}$ are small, inter-group inequalities in skill for the national sector, net earnings, and consumption are lowest at $s=0$, but the inequality in skill for the local sector is highest at $s=0$.

(ii) If $T_{N}, T_{2}, \delta_{N}$, and $\delta_{L}$ are sufficiently large, the inter-group inequalities are lowest when $s$ is in the intermediate range.

When the sectoral productivities and the effectiveness of minority education are low, commonlanguage-only education attains lowest inter-group inequalities in skill for the national sector (common language skill), net earnings, and consumption, but it leads to the highest inequality in skill 
for the local sector (mother tongue skill), which is essential skill outside of work for most people. In contrast, bilingual education brings a lower gap in mother tongue skill but at the cost of higher gaps in other dimensions. Balanced dual education achieves lowest inter-group inequalities in all dimensions only when the productivities and the effectiveness of education are sufficiently large.

\section{General case}

This section examines the general case in which some people may not have enough wealth to make optimal educational investment. This case would be relevant to many developing countries in which students must rely on limited family wealth to pay for study materials, commuting cost, uniforms, and supplementary education even when public schools do not charge tuitions. The important difference from the unconstrained case is that individuals choose different levels of educational spending and different sectors depending on their wealth. First, the section examines how the distribution of wealth affects net earnings and consumption of individuals and intra-group inequalities. Then, as the previous section, the section examines the effect of the weight on the two types of education the minority receive on their human capital, net earnings, consumption, and inter-group inequalities. Let the distribution function of wealth (endowment) of group 2 individuals be $F(a)$, which is differentiable for $a>0$ and allows a mass at $a=0$, i.e., $F(0)>0 .^{21}$

\subsection{Group 1}

Because group 1's human capital is same in both sectors, as in the previous section, the wage rates per human capital of the two sectors are equated and everyone is indifferent between the sectors. What is different from the unconstrained case is that those who have wealth $a<e_{1}^{*}$ cannot make optimal investment, i.e., $e=a<e_{1}^{*}$, and thus net earnings differ among individuals with different wealth (except among individuals with $a \geq e_{1}^{*}$ ). As shown in Appendix A, levels of net earnings and consumption of group 1 individuals do not depend on the distribution of wealth.

\subsection{Group 2}

Unlike group 1, the fact that the group 2's human capital production functions are asymmetricwithout education, human capital for the national sector is zero but human capital for the local sector is positive -implies that only individuals with particular level(s) of wealth are indifferent between the sectors. Individuals who have greater wealth and thus spend more on education than those who are indifferent between the sectors have a comparative advantage in the national sector and choose the sector, and individuals with smaller wealth choose the local sector.

To be more accurate, depending on whether $e_{2 L}^{*}=0$ or $e_{2 L}^{*}>0$ and the level(s) of wealth at which the indifference condition holds, five qualitatively distinctive cases could be realized. Figure 4 illustrates how educational and sectoral choices depend on wealth for each case.

As shown in the figure, in Cases 1 and $2, e_{2 L}^{*}=0$ holds and thus those who strictly prefer the local sector, i.e., those with $a<e_{2 N}^{*}$ in Case 1 and those with $a<\widehat{a}_{0}\left(<e_{2 N}^{*}\right)$ in Case 2, do not spend on education, i.e., $e=e_{2 L}^{*}=0$. In Case 1, those with $a \geq e_{2 N}^{*}$ are indifferent between the sectors and those who choose the national (local) sector spend $e=e_{2 N}^{*}(e=0)$; in Case 2, those with $a>\widehat{a}_{0}$ strictly prefer the national sector and spend $e=a$ if $a<e_{2 N}^{*}$ and $e=e_{2 N}^{*}$ otherwise.

In Cases 3, 4, and $5, e_{2 L}^{*}>0$ holds and thus those who strictly prefer the local sector, i.e., those with $a<e_{2 N}^{*}$ in Case 3, those with $a<\widehat{a} \in\left[e_{2 L}^{*}, e_{2 N}^{*}\right)$ in Case 4 , and those with $a<\widetilde{a}\left(<e_{2 L}^{*}\right)$ in Case 5 , choose positive $e .\left(e_{2 N}^{*}>e_{2 L}^{*}\right.$ is proved in Appendix B.) In Cases 3 and 4 , they spend

\footnotetext{
${ }^{21}$ As shown just below, individual outcomes of group 1 do not depend on the distribution of wealth.
} 


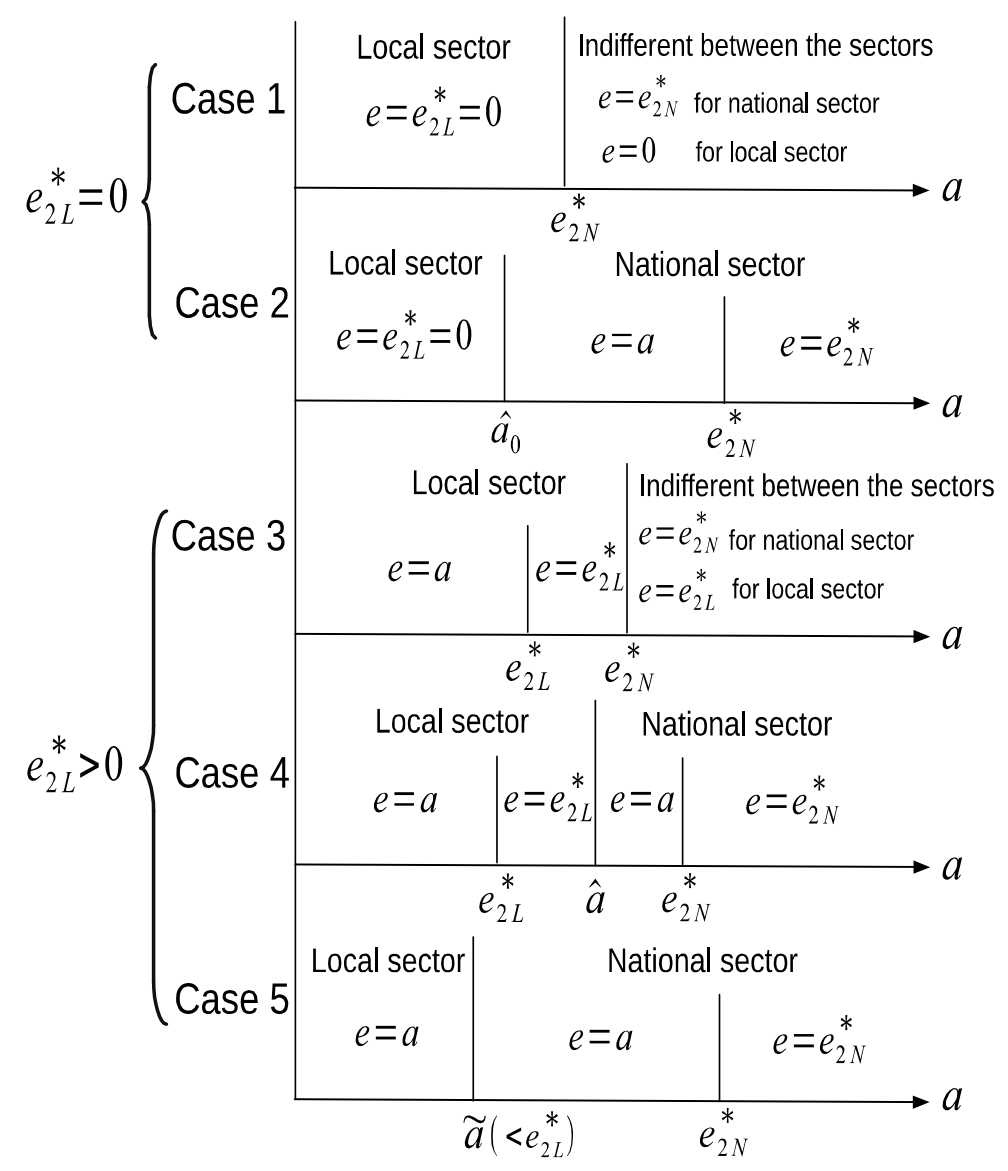

Figure 4: Dependence of educational and sectoral choices of group 2 individuals on wealth

$e=a$ if $a<e_{2 L}^{*}$ and $e=e_{2 L}^{*}$ otherwise, while in Case 5, they spend $e=a$. As for those with wealth greater than the threshold, in Case 3, they are indifferent between the sectors and those who choose the national (local) sector spend $e=e_{2 N}^{*}\left(e=e_{2 L}^{*}\right)$; in Cases 4 and 5, they strictly prefer the national sector and spend $e=a$ if $a<e_{2 N}^{*}$ and $e=e_{2 N}^{*}$ otherwise.

Appendix A explains how the threshold level of wealth and other endogenous variables such as $\frac{H_{2 N}}{H_{2 L}}$, net earnings, and consumption are determined in each case.

\subsection{Results}

Under what conditions is each of the five cases realized? The next lemma shows that it depends on the distribution of wealth and the weight on local language education. Proofs of lemmas and propositions of the general case are contained in Web Appendix C.

Lemma $4(i)$ When $s \leq \underline{s}$ or $s \geq \bar{s}, e_{2 L}^{*}=0$. If the proportion of group 2 individuals with adequate wealth is relatively high (low), i.e., when $F(a)$ is relatively low (high) for given a, Case 1 (Case 2) is realized: the indifference condition holds for individuals with $a \geq e_{2 N}^{*}\left(a=\widehat{a}_{0}<e_{2 N}^{*}\right)$.

(ii) When $s \in(\underline{s}, \bar{s})$, as the proportion of those with adequate wealth becomes lower, the realized equilibrium changes in the following order. ${ }^{22}$

\footnotetext{
${ }^{22}$ Given the distribution of wealth, Case 2 is realized when $s$ is relatively low and high. (As shown in Figure 5, when
} 


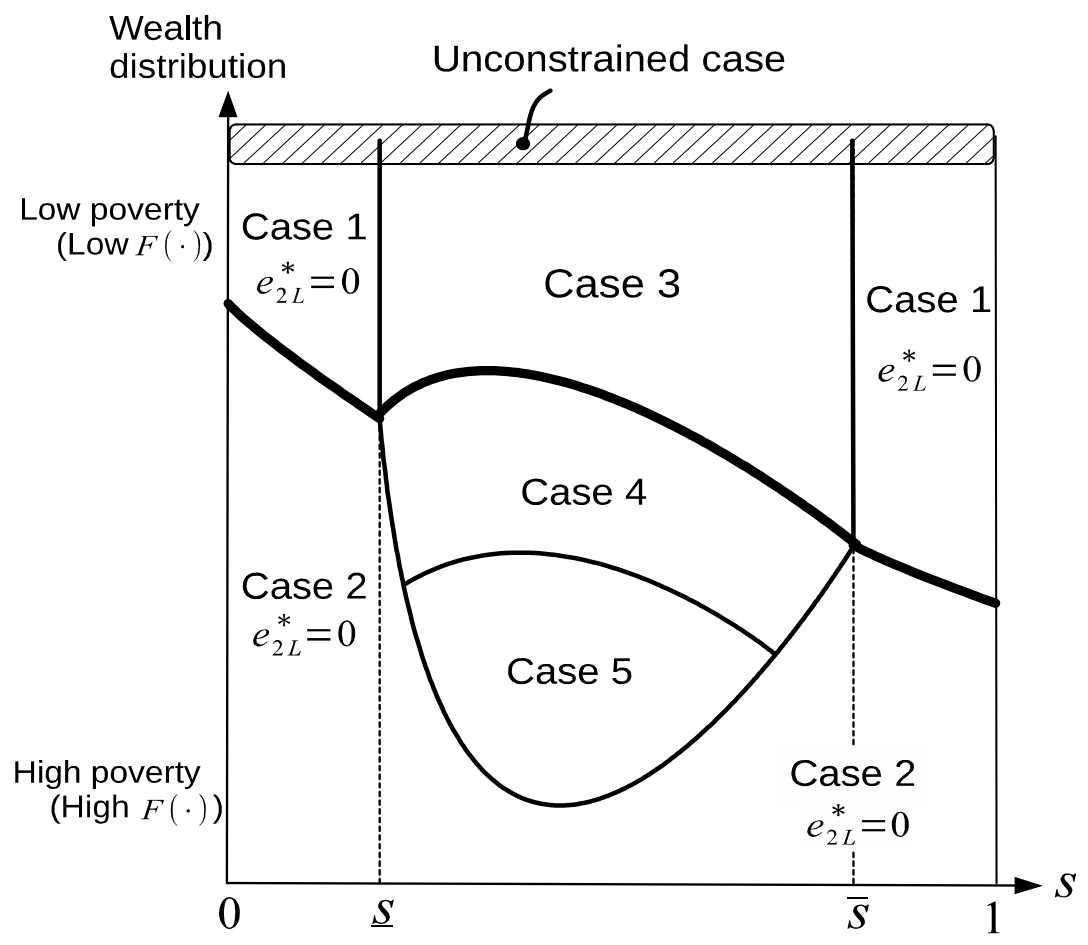

Figure 5: Wealth distribution and realized cases (Lemma 4)

(a) Case 3: $e_{2 L}^{*}>0$ and the indifference condition holds for individuals with $a \geq e_{2 N}^{*}$.

(b) Case 4: $e_{2 L}^{*}>0$ and the indifference condition holds for individuals with $a=\widehat{a} \in\left[e_{2 L}^{*}, e_{2 N}^{*}\right)$.

(c) Case 5, except when $s$ is close to $\underline{s}$ or $\bar{s}: e_{2 L}^{*}>0$ and the indifference condition holds for individuals with $a=\widetilde{a}<e_{2 L}^{*}$.

(d) Case 2: $e_{2 L}^{*}=0$ and the indifference condition holds for individuals with $a=\widehat{a}_{0}<e_{2 N}^{*}$.

Figure 5 illustrates the lemma. When $s \leq \underline{s}$ or $s \geq \bar{s}, e_{2 L}^{*}=0$ and thus Case 1 or Case 2 is realized. Given $s$, Case 1 (Case 2) is realized when the proportion of group 2 individuals with adequate wealth is relatively high (low), i.e., when cumulative distribution $F(a)$ is relatively low (high) for given $a .^{23}$ When $s \in(\underline{s}, \bar{s})$, all the cases except Case 1 could be realized. Given $s$, as the proportion of individuals with adequate wealth becomes lower, i.e., as $F(a)$ for given $a$ becomes higher, the realized equilibrium changes in the following order: Case 3, Case 4, Case 5 (except when $s$ is close to $\underline{s}$ or $\bar{s}$ ), and Case $2 .{ }^{24}$ Note that the unconstrained case examined in

the proportion of those with adequate wealth is extremely low, Case 2 is realized for any $s$.). To be more precise, when $s \in(\underline{s}, \bar{s})$, Case 2 is realized at least for $s \in(\underline{s}, \underline{s}(F))$ and for $s \in(\bar{s}(F), \bar{s})$, where $\underline{s}(F)(\bar{s}(F))$ is critical $s$ below (above) which $e_{2 L}^{*}=0$ holds and $\underline{s}(F)$ increases $(\bar{s}(F)$ decreases) as the proportion of those with adequate wealth falls. (It is not analytically clear if other critical values exist between $\underline{s}(F)$ and $\bar{s}(F)$, but numerical simulations with truncated lognormal distributions suggest other thresholds do not exist.) Case 5 does not exist when $s$ is close to $\underline{s}$ or $\bar{s}$ : as shown in Figure 5, while the dividing line between Case 3 and Case 4 and the one between Case 1 and Case 2 intersect at $s=\underline{s}, \bar{s}$, the one between Case 4 and Case 5 does not intersect with them.

${ }^{23}$ The proof of the proposition also shows that, as illustrated in the figure, given the distribution of wealth, Case 2 (Case 1) is realized when $s$ is low (high), and, as the proportion falls, the region of Case 2 (Case 1) expands (shrinks).

${ }^{24}$ Shapes of the dividing line between Case 3 and Case 4, the one between Case 4 and Case 5 , and ones between Case 2 and Cases 4 and 5 might be different from the figure, although the results below do not depend on shapes of these lines. What is proved analytically about these lines is contained in the lemma and the footnote attached to it. 
the previous section is a special case of Cases 1 and 3, where everyone has enough wealth to make optimal investment, i.e., $a \geq e_{2 N}^{*}>e_{2 L}^{*}$ holds for everyone. ${ }^{25}$

\subsubsection{Effects of the distribution of wealth}

As noted above, the distribution of wealth does not affect net earnings and consumption of group 1 individuals with given levels of wealth. How about group 2 individuals? Since their net earnings and consumption depend on $\frac{H_{2 N}}{H_{2 L}}$, the next lemma examines how wealth distribution affects $\frac{H_{2 N}}{H_{2 L}}$.

\section{Lemma $5^{26}$}

(i) If the proportion of group 2 individuals with adequate wealth is high enough $(F(a)$ for given a is low enough) that Case 1 or Case 3 is realized, a change in the proportion of such individuals does not affect $\frac{H_{2 N}}{H_{2 L}}$.

(ii) Otherwise, as the proportion of those with adequate wealth is lower, $\frac{H_{2 N}}{H_{2 L}}$ becomes smaller.

If the proportion of the minority with adequate wealth is high enough $(F(a)$ for given $a$ is low enough) that Cases 1 or 3 is realized, as with the majority, the distribution of wealth does not affect $\frac{H_{2 N}}{H_{2 L}}$. As the proportion of such individuals becomes lower, a smaller fraction of individuals can financially access the national sector. However, a higher proportion of them, who are indifferent between the sectors, come to choose the sector (footnote 26), which keeps $\frac{H_{2 N}}{H_{2 L}}$ unchanged.

By contrast, if the proportion of those with adequate wealth is not high and thus Cases 2,4 , or 5 is realized, as the proportion of such individuals is lower, $\frac{H_{2 N}}{H_{2 L}}$ becomes smaller. In these cases, those with relatively large wealth uniformly choose the national sector. Hence, a decline in the fraction of people accessible to the national sector leads to a fall in $\frac{H_{2 N}}{H_{2 L}}$, although a decrease in the threshold wealth satisfying the indifference condition (footnote 26) mitigates the decrease of $\frac{H_{2 N}}{H_{2 L}}$.

The result that the distribution of wealth affects $\frac{H_{2 N}}{H_{2 L}}$ in Cases 2, 4, and 5 implies that net earnings, consumption, and human capital of group 2 individuals are influenced by the distribution in these cases. The next proposition shows how the distribution of wealth affects these variables and their inequalities between national and local sector workers.

Proposition 3 Suppose that the proportion of group 2 individuals with adequate wealth falls.

(i) If the proportion of those with adequate wealth is high enough that Cases 1 or 3 is realized, as with to group 1, net earnings, consumption, and human capital of individuals with given wealth do not change. The change in the proportion affects inequalities in these variables only directly.

(ii) Otherwise, net earnings and consumption of those who have relatively large (small) wealth and thus choose the national (local) sector increase (decrease). The decreased proportion raises earnings and consumption disparities between any pairs of national and local sector workers with given levels of wealth.

${ }^{25}$ Which case is realized also depends on sectoral productivities $T_{N}, T_{2}$ and the effectiveness of education $\delta_{N}, \delta_{L}$. When $s$ is low or high enough that $e_{2 L}^{*}=0$, it can be proved that Case 1 (Case 2) is realized when these variables are relatively low (high). When $s$ is intermediate and thus $e_{2 L}^{*}>0$, analytical results are not obtained but numerical simulations suggest that as levels of these variables increase, the realized case changes from Case 3 first to Case 4 , then to Case 5. Intuitively, given wealth distribution, higher values of $T_{N}, T_{2}, \delta_{N}$, or $\delta_{L}$ make educational investment more profitable and raise $e_{2 N}^{*}$ and $e_{2 L}^{*}$, thus a lower proportion of individuals make optimal investment.

${ }^{26}$ The proof of the lemma also shows that, as the proportion of those with adequate wealth becomes lower, a higher fraction of those with $a \geq e_{2 N}^{*}$, who are indifferent between the sectors, choose the national sector in (i), while the threshold wealth satisfying the indifference condition becomes smaller in (ii). 
If the proportion of minority individuals with adequate wealth is high enough that Cases 1 or 3 is realized, as with the majority, a change in the proportion does not affect $\frac{H_{2 N}}{H_{2 L}}$. Thus, net earnings, consumption, and human capital of individuals with given wealth do not change. The change in wealth distribution affects distributions of these variables only directly.

By contrast, if the proportion of such individuals is not high, a decrease in the proportion lowers $\frac{H_{2 N}}{H_{2 L}}$ and thus raises $\frac{w_{2 N}}{P_{2}}$ (lowers $\frac{w_{2 L}}{P_{2}}$ ). As a result, given levels of wealth, net earnings and consumption of those who have relatively large (small) wealth and choose the national (local) sector increase (decrease), and earnings and consumption disparities between any pairs of national and local sector workers rise. $\left[h_{2 N}\left(h_{2 L}\right)\right.$ of those who make optimal investment $e_{2 N}^{*}\left(e_{2 L}^{*}\right)$ increases (decreases) and thus the gap in human capital also rises for some of the pairs.] Hence, a change in the distribution of wealth affects distributions of net earnings, consumption, and human capital not only directly but also indirectly through changes in these variables for given wealth.

This result has implications for within-group inequalities in net earnings and consumption of the two groups. While inequality in wealth of dominant group 1 affects inequalities in net earnings and consumption only directly, inequality in wealth of subordinate group 2 have both direct and indirect effects on the within-group inequalities, as long as the proportion of those with adequate wealth is not high. Hence, a given rise in wealth inequality tends to raise earnings and consumption inequalities of group 2 more than the inequalities of group $1 .{ }^{27}$

Why does the indirect effect exist only for group 2? Unlike the majority, education is a prerequisite for the minority to work in the national sector that requires the skill to use a non-native language to them. As a result, the national sector is "ranked" higher than the local sector only for the minority: group 2 individuals with relatively large wealth for education go to the national sector and those with limited wealth go to the local sector, while the two sectors are indifferent to group 1. A change in wealth distribution alters the proportion of those who can take education sufficient for the national sector and thus $\frac{H_{2 N}}{H_{2 L}}$, wage rates, net earnings and consumption, unless the proportion of such individuals is more than enough for national sector jobs.

\subsubsection{Effects of the weight on mother tongue education}

The remaining part of the subsection examines the question analyzed in the previous section: for group 2 individuals, what is a desirable combination of the two types of education in terms of future net earnings and consumption, and what is a desirable combination in terms of skill development? The important difference from the unconstrained case is that individuals choose different levels of educational spending and different sectors depending on their wealth. Hence, answers to the question could differ among individuals with different wealth.

The next lemma shows that $\frac{H_{2 N}}{H_{2 L}}$ decreases with $s$, as in the unconstrained case.

Lemma $6 \frac{H_{2 N}}{H_{2 L}}$ decreases with $s$.

Hence, an increase in $s$ has a positive (negative) direct effect on net earnings and consumption of local (national) sector workers through human capital, while it has a negative (positive) indirect effect through a fall in $\frac{H_{2 N}}{H_{2 L}}$ and thus a decrease in $\frac{w_{2 L}}{P_{2}}$ (an increase in $\frac{w_{2 N}}{P_{2}}$ ).

The next lemma examines the total effect on net earnings and consumption when $e_{2 L}^{*}=0$ and when $e_{2 L}^{*}>0$ separately.

Lemma 7 (i) Suppose $e_{2 L}^{*}=0$. Then, net earnings and consumption of any group 2 individual decrease with $s$.

\footnotetext{
${ }^{27}$ This claim cannot be proved analytically, but numerical simulations suggest that this is the case.
} 
(ii) Suppose $e_{2 L}^{*}>0$.

(a) If $T_{N}, T_{2}, \delta_{N}$, and $\delta_{L}$ are low, the result is same as $(i) .^{28}$

(b) Otherwise, net earnings and consumption of individuals with very small wealth, who choose the local sector, decrease with $s$, and those of other individuals decrease with s for large $s$. If $T_{N}, T_{2}, \delta_{N}$, and $\delta_{L}$ are sufficiently high, these variables of individuals with relatively large wealth increase with $s$ over some ranges of $s .^{29}$

As with the unconstrained case, net earnings and consumption of the minority decrease with $s$ when $e_{2 L}^{*}=0$ and when $e_{2 L}^{*}>0$ and $T_{N}, T_{2}, \delta_{N}$, and $\delta_{L}$ are low. By contrast, when $e_{2 L}^{*}>0$ and $T_{N}, T_{2}, \delta_{N}$, and $\delta_{L}$ are not low, the result is different depending on wealth. Net earnings and consumption of those with very small wealth decrease with $s$ for any $s$. As for those with greater wealth, these variables decrease with $s$ for large $s$, and if $T_{N}, T_{2}, \delta_{N}$, and $\delta_{L}$ are sufficiently high, the variables of those with relatively large wealth increase with $s$ over some ranges of $s$.

The result for those with very small wealth, who choose the local sector, can be explained as follows. An increase in $s$ has a positive direct effect on net earnings and consumption of local sector workers through $h_{2 L}$, while it has a negative indirect effect through falls in $\frac{H_{2 N}}{H_{2 L}}$ and thus $\frac{w_{2 L}}{P_{2}}$. The positive direct effect increases with wealth, because an individual with greater wealth spends more on education and thus benefits more from the increased weight on mother tongue education. When she has little wealth to spend on education, the positive effect is small and is dominated by the negative effect for any $s$, thus net earnings and consumption always decrease with $s$.

Based on the lemma, the next proposition examines the effect of $s$ on net earnings and consumption by taking into account how $s$ affects whether $e_{2 L}^{*}=0$ or $e_{2 L}^{*}>0$. Thereafter, it is assumed that $\bar{l}$ is not very large compared to wealth levels of most individuals. ${ }^{30}$

\section{Proposition 4}

(i) If $T_{N}, T_{2}, \delta_{N}$, and $\delta_{L}$ are low or if the proportion of those with adequate wealth is very low, net earnings and consumption of any group 2 individual decrease with $s$ and are highest at $s=0$.

(ii) Otherwise, these variables decrease with $s$ for small $s$ and large $s$.

(a) If $T_{N}, T_{2}, \delta_{N}$, and $\delta_{L}$ are sufficiently high, net earnings and consumption of those with relatively large wealth increase with $s$ over some ranges of intermediate $s$. Further, if $T_{N}, T_{2}$, $\delta_{N}$, and $\delta_{L}$ are high enough, these variables are highest at intermediate $s$, where s maximizing the variables of local sector workers weakly increases with a.

(b) Net earnings and consumption of those with very small wealth, who choose the local sector, decrease with $s$ and are highest at $s=0$.

As in the unconstrained case, if $T_{N}, T_{2}, \delta_{N}$, and $\delta_{L}$ are low, net earnings and consumption of the minority decrease with $s$ and are highest at $s=0$. This is also the case if the proportion of those with adequate wealth is very low and thus Case 2 is realized for any $s$ (Figure 5).

\footnotetext{
${ }^{28}$ As for those who have relatively large wealth and thus choose the national sector in Case 4, the result is proved analytically only for large $s$, but numerical simulations suggest that the result holds for any $s$.

${ }^{29}$ In Cases 4 and 5 , the result is not proved analytically for those who choose the local sector and for $\delta_{L}$, but numerical exercises suggest that the result holds for these workers with relatively large $a$ and for high enough $\delta_{L}$.

${ }^{30}$ The assumption is needed to prove the existence of intermediate $s$ maximizing net earnings and consumption in (ii)(a) of the next proposition and intermediate $s$ maximizing $h_{2 N}$ in (ii)(a) of Proposition 5 below. Numerical simulations suggest that, when $\frac{a}{\bar{l}}$ is low for many individuals and $\delta_{L}$ is small, irrespective of levels of $T_{N}, T_{2}$, and $\delta_{N}$, net earnings and consumption (or $h_{2 N}$ ) of any individual decrease with $s$ and thus they are highest at $s=0$. In contrast, if $\delta_{L}$ is sufficiently large, these variables are highest at intermediate $s$ even when $\frac{a}{\bar{l}}$ is low for many.
} 


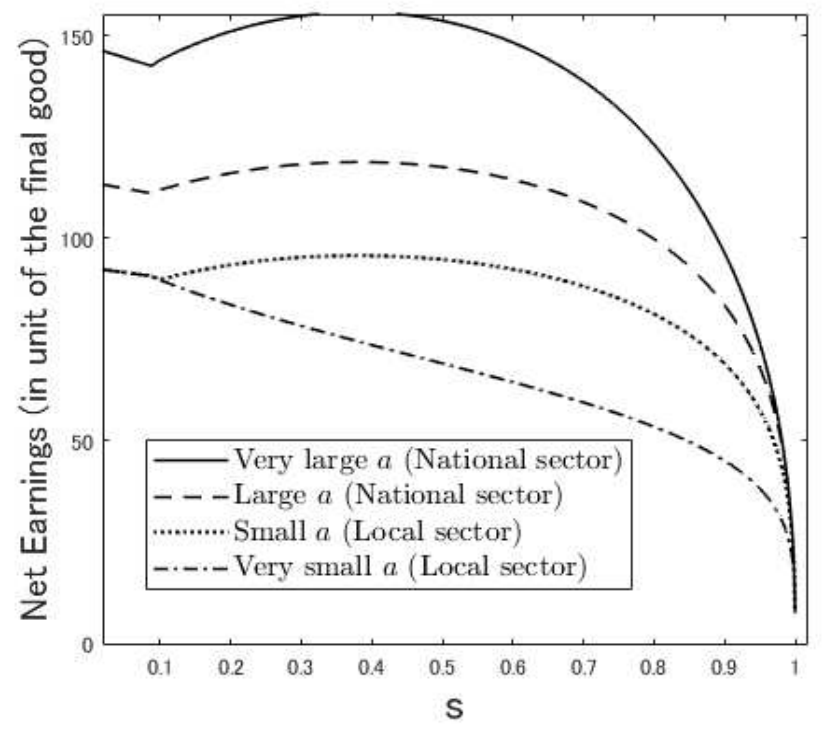

(a) Relatively high $T_{N}, T_{2}, \delta_{N}$, and $\delta_{L}$

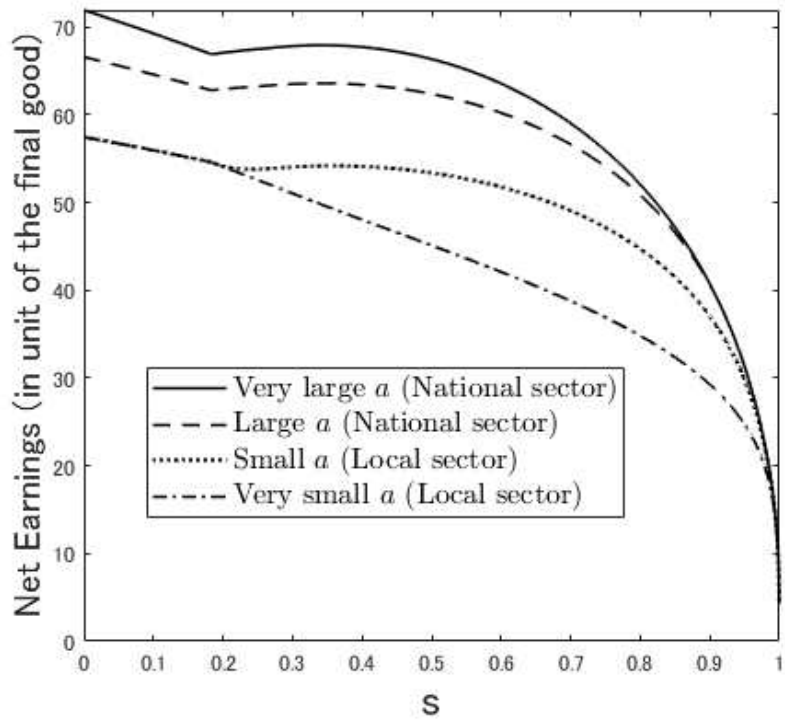

(b) Relatively low $T_{N}, T_{2}, \delta_{N}$, and $\delta_{L}$

Figure 6: Numerical example of the relationship between $s$ and net earnings (in unit of the final good) (Proposition 4)

Otherwise, the result is different depending on wealth. Net earnings and consumption of those with very small wealth, who choose the local sector, decrease with $s$ and are maximized under common-language-only education. These variables of those with greater wealth decrease with $s$ for small $s$ and large $s$ (at least when $e_{2 L}^{*}=0$ ), while for intermediate $s$, the relationship with $s$ is not analytically clear. However, as for those with relatively large wealth, it can be proved that the variables increase with $s$ over some range of intermediate $s$, if $T_{N}, T_{2}, \delta_{N}$, and $\delta_{L}$ are sufficiently high. Further, if the exogenous variables are high enough, their net earnings and consumption are highest at intermediate $s$. In such case, $s$ maximizing the variables of local sector workers weakly increases with their wealth. That is, as their wealth is higher, a greater weight on mother tongue education is economically optimal. This is because an individual with greater wealth spends more on education and thus benefits more from the increased emphasis on local language education.

Since the sectoral productivities and the effectiveness of education of a country are closely related to the degree of development, the result implies that, in general, if the level of development is low, net earnings and consumption are maximized under common-language-only education; while if the level of development is sufficiently high, they are maximized under bilingual education except for the very poor. As mentioned in the previous section, this is consistent with empirical findings (Angrist and Lavy, 1997; Cappellari and Di Paolo, 2018). The difference in the productivity of education is very large even among developing countries (Singh, 2020). The result implies that if a country has ineffective education system, common-language-only education is economically optimal, otherwise, bilingual education is optimal except for the very poor.

Figure 6 presents a numerical example of the relationship between $s$ and net earnings of those whose wealth is very large, large, small, and very small, (a) when $T_{N}, T_{2}, \delta_{N}$, and $\delta_{L}$ are relatively high and (b) when they are relatively low. Net earnings of those with very small $a$ decrease with $s$, while, as in the unconstrained case (with not very low $T_{N}, T_{2}, \delta_{N}$, and $\delta_{L}$ ), net earnings of the 


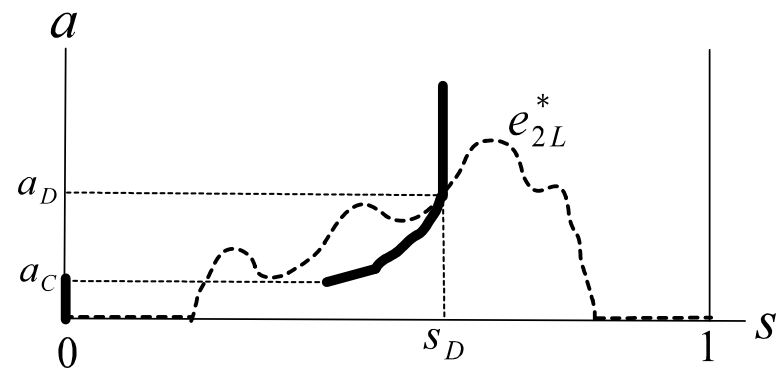

Figure 7: Relationship between wealth and $s$ maximizing net earnings and consumption of local sector workers (Proposition 4 (ii))

other individuals decrease with $s$ for small $s$ and large $s$, and increase with $s$ for intermediate $s$. Their net earnings are highest at intermediate $s$ in (a) and at $s=0$ in (b). ${ }^{31}$

Figure 7 illustrates the relationship between wealth and $s$ maximizing net earnings and consumption of local sector workers, which is represented by a thick solid line, when $T_{N}, T_{2}, \delta_{N}$, and $\delta_{L}$ are high enough. ${ }^{32} s_{D}$ is $s$ maximizing these variables when educational spending is not constrained by wealth. When $a \geq a_{D}$, individuals can make optimal investment $e=e_{2 L}^{*}$ at $s=s_{D}$ and thus the variables are maximized at $s=s_{D}$. When $a \in\left[a_{C}, a_{D}\right)$ and thus $e=e_{2 L}^{*}$ cannot be chosen at $s=s_{D}, e=a<e_{2 L}^{*}$ and $s$ maximizing the variables increases with wealth. When $a<a_{C}$, the variables are maximized at $s=0$ and $e=e_{2 L}^{*}=0$.

The next proposition examines the effect of $s$ on human capital for the national sector and human capital for the local sector.

\section{Proposition 5}

(i) (a) If the proportion of those with enough wealth is very low, $e_{2 L}^{*}=0$ and $h_{2 L}=(\bar{l})^{\gamma}$ for any $s$.

(b) Otherwise, $h_{2 L}$ is highest at intermediate $s$, where $s$ maximizing $h_{2 L}$ weakly decreases with a.

(ii) (a) If $T_{N}, T_{2}, \delta_{N}$, and $\delta_{L}$ are low or if the proportion of those with adequate wealth is very low, $h_{2 N}$ decreases with $s$ and is maximized at $s=0$.

(b) Otherwise, $h_{2 N}$ of those with relatively small wealth decreases with $s$ and is maximized at $s=0$. $h_{2 N}$ of those with greater wealth decreases with $s$ when $s$ is very low or very high (at least when $\left.e_{2 L}^{*}=0\right)$. Their human capital is maximized at $s=0$ if $T_{N}, T_{2}, \delta_{N}$, and $\delta_{L}$ are sufficiently low; otherwise, it is highest at intermediate $s$, where optimal $s$ weakly increases with a.

As in the unconstrained case, human capital for the local sector is highest at intermediate $s$, unless the proportion of those with adequate wealth for education is very low and thus Case 2 is realized for any $s$ (Figure 5), in which case $e_{2 L}^{*}=0$ and thus $h_{2 L}$ is at the lowest level for any $s . s$ maximizing $h_{2 L}$ weakly decreases with $a$. That is, as their wealth increases, a smaller weight on mother tongue education becomes optimal in terms of skill development, which is the opposite from the result on net earnings and consumption of the previous proposition.

\footnotetext{
${ }^{31}$ In both (a) and (b), $\alpha=0.5, \gamma=0.45, \bar{l}=90$, the distribution of wealth follows truncated log normal with maximum 200, mean 20 and variance 80 , and $a=100,30,15$, and 1 for the four types of individuals. In (a), $T_{N}=T_{2}=30, \delta_{N}=15$, and $\delta_{L}=25$, while in (b), $T_{N}=T_{2}=15, \delta_{N}=10$, and $\delta_{L}=20$. In the example, those with $a=100$ and 30 choose the national sector for any $s$ and those with smaller $a$ choose the local sector for any $s$ (except $s$ very close to 1 for those with $a=15$ ).

${ }^{32} s_{D}$ is located in the region in which $e_{2 L}^{*}$ increases with $s$. As in the figure, one cannot rule out the possibility that there exist multiple values of $s$ maximizing $e_{2 L}^{*}$ locally, although simulations suggest that such $s$ is unique.
} 


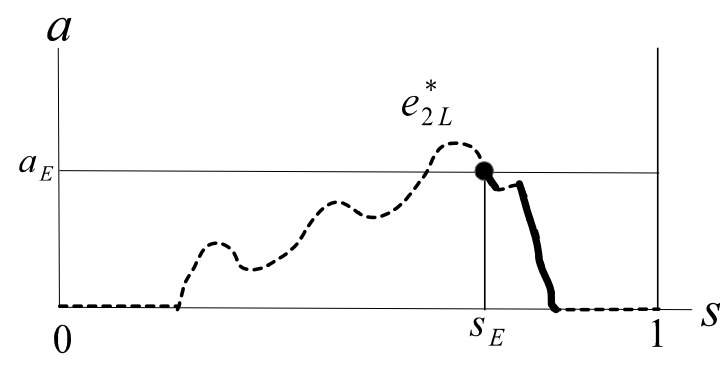

(a) Human capital for the local sector

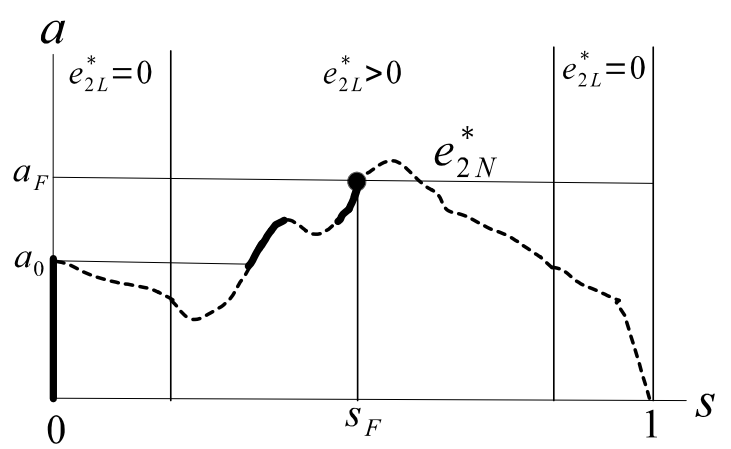

(b) Human capital for the national sector when $T_{N}, T_{2}, \delta_{N}$, and $\delta_{L}$ are high enough

Figure 8: Relationship between $s$ and human capital for each sector (Proposition 5)

As for human capital for the national sector, as in the unconstrained case, if $T_{N}, T_{2}, \delta_{N}$, and $\delta_{L}$ are low, it decreases with $s$ and is maximized at $s=0$. This is also the case if the proportion of individuals with adequate wealth is very low. Otherwise, the result depends on wealth. Human capital of those with relatively small wealth decreases with $s$ and is maximized at $s=0$. As for those with greater wealth, if $T_{N}, T_{2}, \delta_{N}$, and $\delta_{L}$ are sufficiently low, $h_{2 N}$ is highest at $s=0$; otherwise, $h_{2 N}$ is highest at intermediate $s$, where $s$ maximizing the human capital weakly increases with $a$. That is, as their wealth increases, a greater weight on mother tongue education becomes desirable for skill development, which is the opposite from the result on human capital for the local sector.

Figure 8 (a) illustrates the relationship between wealth and $s$ maximizing human capital for the local sector. ${ }^{33} s_{E}$ is $s$ maximizing $h_{2 L}$ when educational spending is not constrained by wealth. When $a \geq a_{E}$, individuals make optimal investment $e=e_{2 L}^{*}$ at $s=s_{E}$ and thus $h_{2 L}$ is maximized at $s=s_{E}$. When $a<a_{E}$ and thus $e=e_{2 L}^{*}$ cannot be chosen at $s=s_{E}, s$ maximizing $h_{2 L}$ is on a portion of the thick solid curve (a segment of the $e_{2 L}^{*}$ profile) below the wealth level, which implies that such $s$ weakly decreases with $a .^{34}$

Figure 8 (b) illustrates the relationship between wealth and $s$ maximizing human capital for the national sector, when $T_{N}, T_{2}, \delta_{N}$, and $\delta_{L}$ are high enough. ${ }^{35} s_{F}$ is $s$ maximizing $h_{2 N}$ when educational spending is not constrained by wealth, which is optimal when $a \geq a_{F}$. When $a \in$ $\left[a_{0}, a_{F}\right)$ and thus $e=e_{2 N}^{*}$ cannot be chosen at $s=s_{F}, s$ maximizing $h_{2 N}$ is on a portion of the thick solid curve (a segment of the $e_{2 N}^{*}$ profile) below the wealth level, which implies that such $s$ weakly increases with $a$. When $a<a_{0}, s=0$ maximizes $h_{2 N}{ }^{36}$

\footnotetext{
${ }^{33}$ As illustrated in the figure, it can be shown that $s_{E}$ is located in the region in which $e_{2 L}^{*}$ decreases with $s$. As in the figure, one cannot rule out the possibility that there exist multiple values of $s$ maximizing $e_{2 L}^{*}$ locally, although numerical simulations suggest that such $s$ is unique. The same is true for $e_{2 N}^{*}$.

${ }^{34}$ This result can be explained as follows. Given $e=a$, increasing $s$ raises $h_{2 L}=\left(\bar{l}+\delta_{L} s e\right)^{\gamma}$ as long as $e<e_{2 L}^{*}$ holds, which implies that $h_{2 L}$ is maximized at highest $s$ satisfying $e=e_{2 L}^{*}$. As $e=a$ becomes greater, the range of $s$ satisfying $e<e_{2 L}^{*}$ shrinks and thus highest $s$ satisfying $e=e_{2 L}^{*}$ decreases (see the figure). Hence, the optimal $s$ becomes weakly smaller. ("weakly" is because $h_{2 L}$ might be maximized at highest $s$ satisfying $e=e_{2 L}^{*}<a$.)

${ }^{35}$ As illustrated in the figure, it can be shown that $e_{2 N}^{*}$ decreases with $s$ when $s$ is small and large (at least when $\left.e_{2 L}^{*}=0\right)$, and $s_{F}$ is located in a region in which $e_{2 N}^{*}$ increases with $s$.

${ }^{36}$ The result for $a \in\left[a_{0}, a_{F}\right)$ can be explained similarly to the corresponding result for $h_{2 L}$ : given $e=a$, decreasing $s$ raises $h_{2 N}=\left[\delta_{N}(1-s) e\right]^{\gamma}$, as long as $e<e_{2 N}^{*}$ holds; as $e=a$ becomes greater, the range of $s$ satisfying $e<e_{2 N}^{*}$ shrinks and thus the optimal $s$ becomes weakly greater.
} 
The result implies that balanced bilingual education is crucial for skill development of those who go to the local sector subsequently, unless the proportion of those with adequate wealth is very low. By contrast, common-language-only education maximizes human capital for the national sector if an individual has relatively small wealth, if $T_{N}, T_{2}, \delta_{N}$, and $\delta_{L}$ are low, or if the proportion of those with enough wealth is very low. However, for most national sector workers, the mother tongue is a major language at home and thus is important for health, child-rearing, and so forth. Hence, it would be fair to say that the dual education is beneficial for them as well.

Finally, based on the propositions, the following corollary presents the result on inter-group inequalities in human capital, net earnings, and consumption.

Corollary 2 Suppose that $T_{1} \geq T_{2}$.

(i) If $T_{N}, T_{2}, \delta_{N}$, and $\delta_{L}$ are low or if the proportion of individuals with adequate wealth is very low, inter-group inequalities in human capital for the national sector, net earnings, and consumption are lowest but the inequality in human capital for the local sector is highest at $s=0$.

(ii) Otherwise, if $T_{N}, T_{2}, \delta_{N}$, and $\delta_{L}$ are sufficiently high, the inter-group inequalities are lowest when $s$ is in the intermediate range, except inequalities in net earnings and consumption of the very poor and in human capital for the national sector of those with relatively small wealth, which are lowest at $s=0$.

If $T_{N}, T_{2}, \delta_{N}$, and $\delta_{L}$ are low or if the proportion of those with adequate wealth is very low, exclusively common language education minimizes inter-group inequalities in net earnings, consumption, and human capital for the national sector, but it maximizes the inequality in human capital for the local sector. Otherwise, if the sectoral productivities and the effectiveness of education are sufficiently high, balanced allocation of the educational budget to the two types of education minimizes inter-group inequalities in most dimensions, but the inequalities in net earnings and consumption of the very poor and in human capital for the national sector of those with relatively small wealth are lowest under common-language-only education.

\section{$5 \quad$ Policy Implications}

\subsection{Implications for language education}

The result suggests that balanced bilingual education is important of skill development of minority individuals: Proposition 5 shows that it is crucial for those who go to the local sector; ${ }^{37}$ while the proposition shows that common-language-only education maximizes human capital for the national sector under certain conditions, considering that mother tongue skill is important for child-rearing, health, and so on for most national sector workers, the dual education is beneficial for them too. This is mostly consistent with a general consensus among specialists on language and education (Ball, 2011; Heugh, 2011) and findings in economics (Jain, 2017; Ramachandran, 2017).

In contrast, according to Proposition 4, while dual education maximizes net earnings and consumption of most minority people when sectoral productivities and the effectiveness of education are sufficiently high, common-language-only education always maximizes these variables of the very poor and when the productivities and the effectiveness of education are low (or when the proportion of those with adequate wealth is very low), maximizes the variables of everyone. The result implies that, in general, when the level of development of a country is low, net earnings and consumption are highest under common-language-only education; when the level of development is sufficiently

\footnotetext{
${ }^{37}$ As shown in the proposition, the optimal weight on the two types of education differs depending on individual wealth. The same is true for the result on net earnings and consumption below.
} 
high, they are highest under bilingual education except for the very poor, which is consistent with empirical findings (Angrist and Lavy, 1997; Cappellari and Di Paolo, 2018).

These results suggest that policies that bring good educational and economic outcomes to every minority individual differs depending on conditions or the level of development of the country.

When the conditions are favorable (the effectiveness of education and the sectoral productivities are sufficiently high, and the proportion of those with adequate wealth is not very low), which is likely to the case when the country's level of development is not low, the government should implement bilingual education together with redistributive policies that support educational investment of individuals with little wealth, such as income transfers, tuition subsidy, and education loans. ${ }^{38}$ Without the latter policies, the very poor lose economically from the implementation of the dual education, because they cannot spend sufficiently enough on education to benefit from it.

By contrast, when the country has unfavorable conditions, which is likely to be true when the level of development is low, bilingual education is better than common-language-only education in terms of skill development, but is worse in terms of consumption. Considering that mother tongue skill, which is important at home even for most national sector workers, is lowest under common-language-only education, ideally, what the government should conduct in the first place is policies changing the adverse conditions: improving sectoral productivities and the effectiveness of education. If these policies are conducted on a sufficient scale, the dual education coupled with redistribution towards the very poor can bring good outcomes to all.

The government, however, may not be able to implement these policies on a sufficient scale for budgetary or other reasons. If this is the case, the government faces a difficult choice between consumption and skill development. If the government highly prioritizes the economic outcome, common-language-only education might be conducted at the expense of mother tongue skill; otherwise, bilingual education might be conducted at the cost of consumption.

The propositions also imply that introducing mother tongue education on a small scale, i.e., $s$ small enough that $e_{2 L}^{*}=0$ holds, is definitely worse than common-language-only education: it does not improve mother tongue skill and lowers common language skill and consumption of all. The range of $s$ satisfying $e_{2 L}^{*}=0$ is wide when conditions of the country are not good, which is shown for the unconstrained case in Lemma $1 .^{39}$ This implies that, if the government introduces bilingual education under the unfavorable conditions, the weight on mother tongue education must be sufficiently high, although, as mentioned above, such policy costs consumption.

Note that the model does not take into account several important effects of the choice of language in education. Mother tongue education would raise ethnic language skill and contribute to the accumulation of social capital in the local ethnic community. It might also stimulate political participation and increase support for democracy (Albaugh, 2016). Common language education, on the other hand, would help people identify with the nation and contribute to national unity and stability. It might also reduce linguistic diversity and promote public goods provision and economic growth (Desmet, Ortuño-Ortín, and Wacziarg, 2012). Policy implementation in the actual society needs to take into account these effects as well as the effects considered in the model.

\footnotetext{
${ }^{38}$ Of course, given weights on the two types of education (given $s$ ), redistribution toward wealth constrained individuals would be desirable, because the policies raise their and aggregate consumption. Rather, what the statement in the main text asserts is that redistribution of a sufficient scale toward the very poor is needed to implement the dual education (to choose intermediate $s$ ).

${ }^{39}$ As for the general case, this is shown for the condition on the proportion of those with adequate wealth in footnote 22 attached to Lemma 4 (Figure 5) and can be shown for other conditions using the equation in the proof of Lemma 1, and Lemmas A1 and A2 in the proof of Lemma 7.
} 


\subsection{Implications for between-group and within-group inequalities}

Since the education policy of the minority does not affect educational and economic outcomes of the majority, the above results have direct implications for inter-group inequalities in skill and consumption. That is, if conditions of the country are favorable or the level of development is not low, dual education coupled with redistributive policies towards the very poor can reduce the inequalities; otherwise, unfavorable conditions must be changed to reduce the gap in consumption. When the conditions cannot be improved sufficiently, bilingual education brings a smaller gap in skill but a greater gap in consumption than common-language-only education.

Proposition 3 has implications for within-group inequalities in net earnings and consumption. While inequality in wealth of dominant group 1 affects inequalities in these variables only directly, inequality in wealth of subordinate group 2 have both direct and indirect effects on the within-group inequalities, as long as the proportion of those with adequate wealth is not high. Thus, a given rise in wealth inequality tends to cause greater increases in earnings and consumption inequalities of group 2 than the inequalities of group 1 .

Hence, redistributive policies that increase access to education of the poor would be more important for group 2 than for group 1: redistribution towards the minority not only lowers intergroup inequalities in skill and consumption but also is more effective in reducing the within-group inequalities.

\section{Conclusion}

Poor economic performance of minority (or subordinate) groups and large economic disparity between these groups and the majority (or dominant) group are major concerns in most countries. The mother tongue of the latter group is the common language in many of these countries. How much weights should be placed on common language education and on ethnic language education is a crucial issue in school education of minority students.

This paper has developed a model to study the issue theoretically. In particular, it has analyzed how weights on the two types of education affect skill, earnings, consumption, and their inter-group inequalities. It also has examined implications of the asymmetric language positions of the groups for sectoral choices and the within-group inequalities. Main results are summarized as follows.

First, regarding skill development of the minority, balanced bilingual education is desirable: it is crucial for those with limited wealth; as for those with greater wealth, common-language-only education could maximize common language skill used in their jobs under certain conditions, but, considering that mother tongue skill is important for off-the-job activities such as child-rearing, the dual education is beneficial for them too.

Second, regarding earnings net of educational expenditure and consumption of the minority, balanced dual education is desirable only when the country has favorable conditions (i.e., sectoral productivities and the effectiveness of education are sufficiently high, and the proportion of those with adequate wealth for education is not very low) and only for those with sufficient wealth. Common-language-only education is always optimal for those with little wealth and, when the country's conditions are bad, it is optimal for all. In the real economy, the conditions are closely related to the level of development of the country. Thus, the result suggests that, in general, if the level of development is low, common-language-only education is desirable in terms of the economic outcomes, otherwise, balanced bilingual education is desirable except for the very poor.

Third, because the majority are not affected by weights on the two types of education the minority receive, the above results directly apply to inter-group inequalities in skill, net earnings, and consumption. For example, switching from exclusively common language education to balanced 
dual education is able to curtail the inter-group inequalities in earnings and consumption (except at the bottom of the distributions) only when the country has favorable conditions.

The results imply that policies that bring good educational and economic outcomes to every minority individual and reduce the inter-group inequalities differ depending on the above-mentioned conditions of the country. If the conditions are favorable, the government should implement balanced bilingual education together with redistributive policies that support educational investment of those with little wealth. Otherwise, it should first conduct policies changing the adverse conditions: improving the productivities and the effectiveness of education. If these policies are conducted on a sufficient scale, dual education coupled with redistribution towards the very poor can bring good outcomes to all. On the other hand, if the government cannot implement these policies on a sufficient scale, it faces a difficult choice between educational and economic outcomes.

Finally, a change in within-group wealth inequality tends to have greater effects on earnings and consumption inequalities of the minority than the inequalities of the majority. This result suggests that redistributive policies that increase access to education of the poor would be more important for the minorty than for the majority.

\section{References}

[1] Albaugh, Ericka A. (2016), "Language, education, and citizenship in Africa", Afrobarometer WP No. 162.

[2] Alesina, Alberto, Stelios Michalopoulos, and Elias Papaioannou (2016), "Ethnic inequality," Journal of Political Economy 124 (2), 428-488.

[3] Angrist, J. and Lavy, V. (1997), "The Effect of a change in language of instruction on the returns to schooling in Morocco," Journal of Labor Economics 15, S48-S76.

[4] Azam, Mehtabul, Aimee Chin, and Nishith Prakash (2013), "The returns to English-language skills in India," Economic Development and Cultural Change 61(2), 335-367.

[5] Ball, Jessica (2011), Enhancing Learning of Children from Diverse Language Backgrounds: Mother Tongue-Based Bilingual or Multilingual Education in the Early Years, Paris, UNESCO.

[6] Benson, Carol, Kathleen Heugh, Berhanu Bogale and Mekonnen Alemu Gebre Yohannes (2012), "Multilingual education in Ethiopian primary schools," in T. Skuttnab-Kangas and K. Heugh eds., Multilingual education and Sustainable Diversity Work. From Periphery to Center, Routledge, London.

[7] Cappellari, Lorenzo and Antonio Di Paolo (2015), "Bilingual Schooling and Earnings: Evidence from a Language-in-Education Reform," IZA Discussion Paper No. 9431.

[8] Chakraborty, Tanika and Shilpi Kapur Bakshi (2016), "English language premium: Evidence from a policy experiment in India," Economics of Education Review 50 (1), 1-16.

[9] Clots-Figueras, I. and P. Masella (2013), "Education, Language and Identity," Economic Journal 123(570), F332-357.

[10] Desmet, K., I. Ortuño-Ortín, and R. Wacziarg (2012), "The Political Economy of Linguistic Cleavages," Journal of Development Economics 97, 322-338.

[11] Faltis, Christian (2014), "Toward a race radical vision of bilingual education for Kurdish users in Turkey: a commentary," Journal of Ethnic and Cultural Studies 1(1), 1-5.

[12] Galor, Oded and Ömer Özak, and Assaf Sarid (2018), "Geographical Origins of Language Structures," available at SSRN: https://ssrn.com/abstract=3097220. 
[13] Gao, Xuesong Andy and Weihong Wang (2017), "Bilingual education in the peoples republic of China," in O. Garcia et al. eds., Bilingual and Multilingual Education, Encyclopedia of Language and Education, Springer, New York.

[14] Ginsburgh, V. A., I. Ortuño-Ortín, and S. Weber (2005), "Disenfranchisement in linguistically diverse societies. The case of the European Union," Journal of the European Economic Association 3(4), 946-965.

[15] Haboud, Marleen and Nicholas Limerick (2017), "Language policy and education in the Andes," in T. L. McCarty and S. May eds., Language Policy and Political Issues in Education, Encyclopedia of Language and Education, Springer, New York.

[16] Heugh, Kathleen (2011), "Theory and practice - Language education models in Africa: research, design, decisionmaking, and outcomes", in A. Ouane and C. Glanz eds., Optimising Learning, Education and Publishing in Africa: The Language Factor, the UNESCO Institute for Lifelong Learning and the Association for the Development of Education in Africa.

[17] Jain, Tarun (2017), "Common Tongue: The Impact of Language on Educational Outcomes", Journal of Economic History 77, 473-510.

[18] Lazear, E. P. (1999), "Culture and language," Journal of Political Economy 107(6), S95-S126.

[19] Melitz, Jacques (2008), "Language and foreign trade," European Economic Review 52(4), $667-699$.

[20] Nguyen, Hoa-Thi-Minh, Tom Kompas, Trevor Breusch, and Michael B. Ward (2017), "Language, Mixed Communes, and Infrastructure: Sources of Inequality and Ethnic Minorities in Vietnam," World Development 96, 145-162.

[21] Nguyen, Xuan Nhat Chi Mai and Van Huy Nguyen (2019), "Language education policy in Vietnam," in A. Liddicoat and R. Kirkpatrick eds., The Routledge international handbook of language education policy in Asia, Routledge, London.

[22] Ortega, J. and T. P. Tangerås (2008), "Unilingual versus bilingual education: A political economy analysis," Journal of the European Economic Association 6(5), 1078-1108.

[23] Pool, Jonathan (1991), "The Official Language Problem," American Political Science Review $85(2), 495-514$.

[24] Ramachandran, Rajesh (2017), "Language use in education and human capital formation: Evidence from the Ethiopian educational reform," World Development 98: 195-213.

[25] Singh, Abhijeet (2020), "Learning More with Every Year: School year productivity and international learning divergence," Journal of the European Economic Association 18(4), 1770-1813.

[26] Tupas, Ruanni and Isabel Pefianco Martin (2017), "Bilingual and mother tongue-based multilingual education in the Philippines," in O. Garcia et al. eds., Bilingual and Multilingual Education, Encyclopedia of Language and Education, Springer, New York.

[27] Vaillancourt, François and François Grin (2000), "Language and Socioeconomic Status in Quebec: Measurement, Findings, Determinants, and Policy Costs, "International Journal of the Sociology of Language 121: 69-92.

[28] Walter, S. L. and Benson, C, (2012), "Language Policy and Medium of Instruction in Formal Education," in B. Spolsky ed., The Cambridge Handbook of Language Policy, Cambridge University Press, Cambridge, pp. 278-300.

[29] Yuki, Kazuhiro (2018), "Is bilingual education desirable in multilingual countries?," MPRA Paper 85034. 


\section{Appendix A Determination of endogenous variables in the general case}

\section{Group 1}

For group 1 individuals with $a \geq e_{1}^{*}$, consumption is (19) as before, while, for individuals with $a<e_{1}^{*}$, from (16) and (15), net earnings in unit of the intermediate good equal

$$
T_{N}(\bar{l}+a)^{\gamma}-\frac{1}{\alpha^{\alpha}(1-\alpha)^{1-\alpha}}\left(\frac{T_{N}}{T_{1}}\right)^{\alpha} a,
$$

and from (15) and the above equation, consumption equals

$$
\begin{aligned}
c_{1}(a) & =\frac{w_{N} h_{1}-P_{1} a}{P_{1}}+a \\
& =\left(\alpha T_{1}\right)^{\alpha}\left((1-\alpha) T_{N}\right)^{1-\alpha}(\bar{l}+a)^{\gamma} .
\end{aligned}
$$

Net earnings in unit of the final good equal consumption minus wealth.

\section{Group 2}

Net earnings in unit of the final good equal consumption minus wealth.

(I) When $e_{2 L}^{*}=0$

Case 1: Indifference condition holds for $a \geq e_{2 N}^{*}$. When $e_{2 L}^{*}=0$ and those with $a \geq e_{2 N}^{*}$ are indifferent between choosing the national sector by expending $e=e_{2 N}^{*}$ on education and choosing the local sector by expending $e=e_{2 L}^{*}=0$, the indifference condition is (27), same as when everyone has enough wealth for education and $e_{2 L}^{*}=0$. Because those with $a<e_{2 N}^{*}$ expend $e=0$ and choose the local sector, $H_{2 N}=\left[\delta_{N}(1-s) e_{2 N}^{*}\right]^{\gamma} p_{2 N}\left(1-F\left(e_{2 N}^{*}\right)\right) L_{2}\left(L_{2}\right.$ is the group 2 population) and $H_{2 L}=(\bar{l})^{\gamma}\left[\left(1-p_{2 N}\right)\left(1-F\left(e_{2 N}^{*}\right)\right)+F\left(e_{2 N}^{*}\right)\right] L_{2}$, where $p_{2 N}$ is the proportion of those with $a \geq e_{2 N}^{*}$ choosing the national sector. Hence,

$$
\frac{H_{2 N}}{H_{2 L}}=\frac{\left[\delta_{N}(1-s) e_{2 N}^{*}\right]^{\gamma} p_{2 N}\left(1-F\left(e_{2 N}^{*}\right)\right)}{(\bar{l})^{\gamma}\left[\left(1-p_{2 N}\right)\left(1-F\left(e_{2 N}^{*}\right)\right)+F\left(e_{2 N}^{*}\right)\right]},
$$

where $e_{2 N}^{*}$ is given by (21).

Once $\frac{H_{2 N}}{H_{2 L}}$ is determined from (28), this equation determines $p_{2 N}$. Since $e=e_{2 L}^{*}=0$ for any individual choosing the local sector, as in the unconstrained case, $c_{2}$ for any $a$ is given by (30).

Case 2: Indifference condition holds for $a=\widehat{a}_{0}<e_{2 N}^{*}$. When $e_{2 L}^{*}=0$ and those with $a=\widehat{a}_{0}<e_{2 N}^{*}$ are indifferent between choosing the national sector by expending $e=\widehat{a}_{0}$ and choosing the local sector without education, the indifference condition is, from (20), (10), and (26),

$$
T_{N}\left(\delta_{N}(1-s) \widehat{a}_{0}\right)^{\gamma}-\frac{1}{1-\alpha}\left(\frac{T_{N} H_{2 N}}{T_{2} H_{2 L}}\right)^{\alpha} \widehat{a}_{0}=\frac{\alpha}{1-\alpha} T_{N} \frac{H_{2 N}}{H_{2 L}}(\bar{l})^{\gamma} .
$$

Since those with $a>\widehat{a}_{0}$ expend $e=\min \left\{a, e_{2 N}^{*}\right\}$ and choose the national sector, and those with $a<\widehat{a}_{0}$ expend $e=0$ and choose the local sector, $H_{2 N}=\left\{\left[\delta_{N}(1-s) e_{2 N}^{*}\right]^{\gamma}\left(1-F\left(e_{2 N}^{*}\right)\right)+\int_{\widehat{a}_{0}}^{e_{2 N}^{*}}\left[\delta_{N}(1-s) a\right]^{\gamma} d F(a)\right\} L_{2}$ and $H_{2 L}=(\bar{l})^{\gamma} F\left(\widehat{a}_{0}\right) L_{2}$. Hence, 


$$
\frac{H_{2 N}}{H_{2 L}}=\frac{\left[\delta_{N}(1-s)\right]^{\gamma}\left[\left(e_{2 N}^{*}\right)^{\gamma}\left(1-F\left(e_{2 N}^{*}\right)\right)+\int_{\widehat{a}_{0}}^{e_{2 N}^{*}}(a)^{\gamma} d F(a)\right]}{(\bar{l})^{\gamma} F\left(\widehat{a}_{0}\right)},
$$

where $e_{2 N}^{*}$ is given by $(21)$.

$\frac{H_{2 N}}{H_{2 L}}$ and $\widehat{a}_{0}$ are obtained by solving (A4) and (A5), which implies that, unlike the previous case, $\frac{H_{2 N}}{H_{2 L}}$ (and thus individual net earnings and consumption) depends on the distribution of wealth.

Finally, $c_{2}$ for $a \geq e_{2 N}^{*}$ is given by (30) as before, while $c_{2}$ for $a \in\left[\widehat{a}_{0}, e_{2 N}^{*}\right)$ is given by, from (20) and (10),

$$
\begin{aligned}
c_{2 N}(a) & =\frac{w_{N} h_{2 N}-P_{2} a}{P_{2}}+a \\
& =(1-\alpha) T_{2}{ }^{\alpha} T_{N}{ }^{1-\alpha}\left(\frac{H_{2 N}}{H_{2 L}}\right)^{-\alpha}\left(\delta_{N}(1-s) a\right)^{\gamma},
\end{aligned}
$$

and $c_{2}$ for $a<\widehat{a}_{0}$ equals, from (26), (9), and (10),

$$
\begin{aligned}
c_{2 L}(a) & =\frac{w_{2 L} h_{2 L}}{P_{2}}+a \\
& =\alpha T_{2}{ }^{\alpha} T_{N}{ }^{1-\alpha}\left(\frac{H_{2 N}}{H_{2 L}}\right)^{1-\alpha}(\bar{l})^{\gamma}+a .
\end{aligned}
$$

(II) When $e_{2 L}^{*}>0$

Case 3: Indifference condition holds for $a \geq e_{2 N}^{*}$. When $e_{2 L}^{*}>0$ and those with $a \geq e_{2 N}^{*}$ are indifferent between choosing the national sector by spending $e=e_{2 N}^{*}$ and choosing the local sector by spending $e=e_{2 L}^{*}$, the indifference condition is (29), same as the unconstrained case. Since those with $a<e_{2 N}^{*}$ spend $e=\min \left\{a, e_{2 L}^{*}\right\}$ and choose the local sector, ${ }^{40} H_{2 N}=\left[\delta_{N}(1-s) e_{2 N}^{*}\right]^{\gamma} p_{2 N}\left(1-F\left(e_{2 N}^{*}\right)\right) L_{2}$

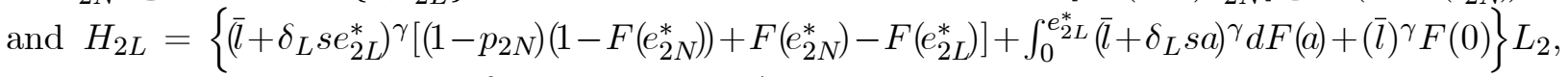
where $p_{2 N}$ is the proportion of those with $a \geq e_{2 N}^{*}$ choosing the national sector. Hence,

$$
\frac{H_{2 N}}{H_{2 L}}=\frac{\left[\delta_{N}(1-s) e_{2 N}^{*}\right]^{\gamma} p_{2 N}\left(1-F\left(e_{2 N}^{*}\right)\right)}{\left(\bar{l}+\delta_{L} s e_{2 L}^{*}\right)^{\gamma}\left[\left(1-p_{2 N}\right)\left(1-F\left(e_{2 N}^{*}\right)\right)+F\left(e_{2 N}^{*}\right)-F\left(e_{2 L}^{*}\right)\right]+\int_{0}^{e_{2 L}^{*}}\left(\bar{l}+\delta_{L} s a\right)^{\gamma} d F(a)+(\bar{l})^{\gamma} F(0)},
$$

where $e_{2 N}^{*}$ and $e_{2 L}^{*}$ are given by (21) and (24), respectively.

Once $\frac{H_{2 N}}{H_{2 L}}$ is determined from (29), the above equation determines $p_{2 N}$.

$c_{2}$ for $a \geq e_{2 L}^{*}$ is given by (30) from (29), while $c_{2}$ for $a<e_{2 L}^{*}$ equals, from (23), (9), and (10),

$$
\begin{aligned}
c_{2 L}(a) & =\frac{w_{2 L} h_{2 L}-P_{2} a}{P_{2}}+a \\
& =\alpha T_{2}^{\alpha} T_{N}{ }^{1-\alpha}\left(\frac{H_{2 N}}{H_{2 L}}\right)^{1-\alpha}\left(\bar{l}+\delta_{L} s a\right)^{\gamma} .
\end{aligned}
$$

Case 4: Indifference condition holds for $a=\widehat{a} \in\left[e_{2 L}^{*}, e_{2 N}^{*}\right)$. When those with $a=\widehat{a} \in$ $\left[e_{2 L}^{*}, e_{2 N}^{*}\right)$ are indifferent between choosing the national sector with $e=\widehat{a}$ and choosing the local sector with $e=e_{2 L}^{*}>0$, the indifference condition is, from (20), (10), and (25),

\footnotetext{
${ }^{40}$ This is obvious for $a \in\left[e_{2 L}^{*}, e_{2 N}^{*}\right)$. The result for $a<e_{2 L}^{*}$ can be proved as follows. Since $w_{2 L} h_{2 L}-P_{2} a>$ $w_{N} h_{2 N}-P_{2} a \Leftrightarrow w_{2 L}\left(\bar{l}+\delta_{L} s a\right)^{\gamma}>w_{N}\left(\delta_{N}(1-s) a\right)^{\gamma}$ holds for those with $a=0$ and with $a=e_{2 L}^{*}$, if there exist ranges of $a \in\left(0, e_{2 L}^{*}\right)$ over which $w_{2 L} h_{2 L}-P_{2} a<w_{N} h_{2 N}-P_{2} a$ is true, there must exist at least two values of $a$ satisfying $w_{2 L}\left(\bar{l}+\delta_{L} s a\right)^{\gamma}=w_{N}\left(\delta_{N}(1-s) a\right)^{\gamma}$, which is not possible.
} 
$T_{N}\left(\delta_{N}(1-s) \widehat{a}\right)^{\gamma}-\frac{1}{1-\alpha}\left(\frac{T_{N} H_{2 N}}{T_{2} H_{2 L}}\right)^{\alpha} \widehat{a}=\frac{1}{1-\alpha}\left(\frac{T_{N} H_{2 N}}{T_{2} H_{2 L}}\right)^{\alpha}\left\{(1-\gamma)\left[\left(\gamma \delta_{L} s\right)^{\gamma} \alpha T_{2}{ }^{\alpha} T_{N}{ }^{1-\alpha}\left(\frac{H_{2 N}}{H_{2 L}}\right)^{1-\alpha}\right]^{\frac{1}{1-\gamma}}+\frac{\bar{l}}{\delta_{L} s}\right\}$.

Since those with $a>\widehat{a}$ choose $e=\min \left\{a, e_{2 N}^{*}\right\}$ and the national sector and those with $a<\widehat{a}$ choose $e=\min \left\{a, e_{2 L}^{*}\right\}$ and the local sector, $H_{2 N}=\left\{\left[\delta_{N}(1-s) e_{2 N}^{*}\right]^{\gamma}\left(1-F\left(e_{2 N}^{*}\right)\right)+\int_{\widehat{a}}^{e_{2 N}^{*}}\left[\delta_{N}(1-s) a\right]^{\gamma} d F(a)\right\} L_{2}$ and $H_{2 L}=\left\{\left(\bar{l}+\delta_{L} s e_{2 L}^{*}\right)^{\gamma}\left(F(\widehat{a})-F\left(e_{2 L}^{*}\right)\right)+\int_{0}^{e_{2 L}^{*}}\left(\bar{l}+\delta_{L} s a\right)^{\gamma} d F(a)+(\bar{l})^{\gamma} F(0)\right\} L_{2}$. Hence,

$$
\frac{H_{2 N}}{H_{2 L}}=\frac{\left[\delta_{N}(1-s)\right]^{\gamma}\left[\left(e_{2 N}^{*}\right)^{\gamma}\left(1-F\left(e_{2 N}^{*}\right)\right)+\int_{\widehat{a}}^{e_{2 N}^{*}} a^{\gamma} d F(a)\right]}{\left(\bar{l}+\delta_{L} s e_{2 L}^{*}\right)^{\gamma}\left(F(\widehat{a})-F\left(e_{2 L}^{*}\right)\right)+\int_{0}^{e_{2 L}^{*}}\left(\bar{l}+\delta_{L} s a\right)^{\gamma} d F(a)+(\bar{l})^{\gamma} F(0)}
$$

where $e_{2 N}^{*}$ and $e_{2 L}^{*}$ are given by (21) and (24), respectively.

$\frac{H_{2 N}}{H_{2 L}}$ and $\widehat{a}$ are obtained by solving (A10) and (A11), which implies that, unlike the previous case, $\frac{H_{2 N}}{H_{2 L}}$ (and thus individual net earnings and consumption) depends on the distribution of wealth.

$c_{2}$ for $a \geq e_{2 N}^{*}$ is given by (30), $c_{2}$ for $a \in\left[\widehat{a}, e_{2 N}^{*}\right)$ is given by (A6), $c_{2}$ for $a \in\left[e_{2 L}^{*}, \widehat{a}\right)$ equals, from (25) and (10),

$$
\begin{aligned}
c_{2 L}^{*}(a) & =\frac{w_{2 L} h_{2 L}^{*}-P_{2} e_{2 L}^{*}}{P_{2}}+a \\
& =(1-\gamma)\left[\left(\gamma \delta_{L} s\right)^{\gamma} \alpha T_{2}^{\alpha} T_{N}{ }^{1-\alpha}\left(\frac{H_{2 N}}{H_{2 L}}\right)^{1-\alpha}\right]^{\frac{1}{1-\gamma}}+\frac{\bar{l}}{\delta_{L} s}+a,
\end{aligned}
$$

and $c_{2}$ for $a<e_{2 L}^{*}$ is given by (A9).

Case 5: Indifference condition holds for $a=\widetilde{a}<e_{2 L}^{*}$. Finally, when those with $a=\widetilde{a}<e_{2 L}^{*}$ are indifferent between choosing the national sector by expending $e=\widetilde{a}$ and choosing the local sector by expending the same $\widetilde{a}$ on education, the indifference condition is, from (20), (23), and $(9)$,

$$
T_{N}\left(\delta_{N}(1-s) \widetilde{a}\right)^{\gamma}=\frac{\alpha}{1-\alpha} \frac{T_{N} H_{2 N}}{H_{2 L}}\left(\bar{l}+\delta_{L} s \widetilde{a}\right)^{\gamma} \Leftrightarrow \frac{H_{2 N}}{H_{2 L}}=\frac{1-\alpha}{\alpha}\left[\frac{\delta_{N}(1-s) \widetilde{a}}{\bar{l}+\delta_{L} s \widetilde{a}}\right]^{\gamma} .
$$

Since those with $a>\widetilde{a}$ expend $e=\min \left\{a, e_{2 N}^{*}\right\}$ and choose the national sector and those with $a<\widetilde{a}$ expend $e=a$ and choose the local sector, $H_{2 N}=\left\{\left[\delta_{N}(1-s) e_{2 N}^{*}\right]^{\gamma}\left(1-F\left(e_{2 N}^{*}\right)\right)+\int_{\widetilde{a}}^{e_{2 N}^{*}}\left[\delta_{N}(1-s) a\right]^{\gamma} d F(a)\right\} L_{2}$ and $H_{2 L}=\left[\int_{0}^{\widetilde{a}}\left(\bar{l}+\delta_{L} s a\right)^{\gamma} d F(a)+(\bar{l})^{\gamma} F(0)\right] L_{2}$. Hence,

$$
\frac{H_{2 N}}{H_{2 L}}=\frac{\left[\delta_{N}(1-s)\right]^{\gamma}\left[e_{2 N}^{*} \gamma\left(1-F\left(e_{2 N}^{*}\right)\right)+\int_{\widetilde{a}}^{e_{2 N}^{*}} a^{\gamma} d F(a)\right]}{\int_{0}^{\widetilde{a}}\left(\bar{l}+\delta_{L} s a\right)^{\gamma} d F(a)+(\bar{l})^{\gamma} F(0)}
$$

where $e_{2 N}^{*}$ is given by $(21)$.

$\frac{H_{2 N}}{H_{2 L}}$ and $\widetilde{a}$ are obtained by solving (A13) and (A14). $c_{2}$ for $a \geq e_{2 N}^{*}$ is given by (30), $c_{2}$ for $a \in\left[\widetilde{a}, e_{2 N}^{*}\right)$ is given by (A6), and $c_{2}$ for $a<\widetilde{a}$ is given by (A9).

\section{Appendix B Proof of lemmas and propositions of the uncon- strained case}

Proof of Lemma 1. By substituting (4) and (10) into the condition for $e_{2 L}^{*}=0, \gamma \delta_{L} s w_{2 L}(\bar{l})^{\gamma-1}-$ $P_{2} \leq 0$ 


$$
\begin{gathered}
\gamma \delta_{L} s \alpha T_{2}^{\alpha} T_{N}{ }^{1-\alpha}\left(\frac{H_{2 N}}{H_{2 L}}\right)^{1-\alpha}(\bar{l})^{\gamma-1} \leq 1 \\
\Leftrightarrow \gamma \delta_{L} s \alpha T_{2}^{\alpha} T_{N}{ }^{1-\alpha}\left\{\left(\frac{1-\alpha}{\alpha} \frac{1-\gamma}{(\bar{l})^{\gamma}}\right)^{1-\gamma}\left[(1-\alpha) \gamma \delta_{N}(1-s) T_{2}^{\alpha} T_{N}{ }^{1-\alpha}\right]^{\gamma}\right\}^{\frac{1-\alpha}{1-\gamma(1-\alpha)}}(\bar{l})^{\gamma-1} \leq 1 \text { (from } \\
\Leftrightarrow\left(\delta_{L} s\right)^{1-\gamma(1-\alpha)}\left[\delta_{N}(1-s)\right]^{\gamma(1-\alpha)} \gamma(1-\gamma)^{(1-\gamma)(1-\alpha)}\left(\alpha T_{2}\right)^{\alpha}\left((1-\alpha) T_{N}\right)^{1-\alpha}(\bar{l})^{\gamma-1} \leq 1 . \quad \text { (A15) }
\end{gathered}
$$

Denote the higher (lower) $s$ satisfying the above equation with equality by $\bar{s}(\underline{s})[\underline{s}<1-\gamma(1-$ $\alpha)<\bar{s}$, which exists when $T_{2}$ and $T_{N}$ are not extemely low. The lemma is straightforward from the equation.

Proof of Lemma 2. The result is straightforward from (28) when $e_{2 L}^{*}=0$. When $e_{2 L}^{*}>0$, the LHS of (29) decreases with $\frac{H_{2 N}}{H_{2 L}}$ and $s$, and the RHS increases with $\frac{H_{2 N}}{H_{2 L}}$. Further, for $s$ satisfying $e_{2 L}^{*}>0$, the RHS increases with $s$, because the derivative of the expression inside the curly bracket of the RHS with respect to $s$ equals

$$
\frac{1}{s^{2}}\left\{\gamma s\left[\left(\gamma \delta_{L} s\right)^{\gamma} \alpha T_{2}^{\alpha} T_{N}{ }^{1-\alpha}\left(\frac{H_{2 N}}{H_{2 L}}\right)^{1-\alpha}\right]^{\frac{1}{1-\gamma}}-\frac{\bar{l}}{\delta_{L}}\right\}>0,
$$

where the inequality sign is from $e_{2 L}^{*}>0$ and (24). Therefore, an increase in $s$ lowers $\frac{H_{2 N}}{H_{2 L}}$.

Proof of Lemma 3. (i) When $e_{2 L}^{*}=0$, consumption of a group 2 individual with wealth $a$ equals, from (30),

$$
\begin{gathered}
c_{2}^{*}(a)=(1-\gamma)\left\{T_{N}(1-\alpha)\left[\gamma \delta_{N}(1-s)\right]^{\gamma}\left(\frac{T_{N} H_{2 N}}{T_{2} H_{2 L}}\right)^{-\alpha}\right\}^{\frac{1}{1-\gamma}}+a \\
=(1-\gamma)\left((1-\alpha) T_{2}^{\alpha} T_{N}^{1-\alpha}\left[\gamma \delta_{N}(1-s)\right]^{\gamma}\left\{\left(\frac{1-\alpha}{\alpha} \frac{1-\gamma}{(\bar{l})^{\gamma}}\right)^{1-\gamma}\left[(1-\alpha) \gamma \delta_{N}(1-s) T_{2}^{\alpha} T_{N}{ }^{1-\alpha}\right]^{\gamma}\right\}^{\frac{-\alpha}{1-\gamma(1-\alpha)}}\right)^{\frac{1}{1-\gamma}}+a(\text { from }(28)) \\
=(1-\gamma)\left\{(1-\alpha) T_{2}^{\alpha} T_{N}^{1-\alpha}\left[\gamma \delta_{N}(1-s)\right]^{\left.(1-\alpha) \gamma\left[\frac{\alpha}{1-\alpha} \frac{(\bar{l})^{\gamma}}{1-\gamma}\right]^{\alpha}\right\}^{\frac{1}{1-\gamma(1-\alpha)}}+a}\right.
\end{gathered}
$$

Hence, $c_{2}$ when $e_{2 L}^{*}=0$ decreases with $s$. The same is true for net earnings in unit of the final good, because they equal consumption minus wealth.

(ii) Only the proof of the result on the consumption is presented, because net earnings in unit of the final good equal consumption minus wealth.

[Condition for $\left.\frac{d c_{2}}{d s}>(<) 0\right]$ From $(30), \frac{d c_{2}}{d s}$ is proportional to $-\left[\frac{\gamma}{1-s}+\alpha\left(\frac{H_{2 N}}{H_{2 L}}\right)^{-1} \frac{d \frac{H_{2 N}}{H_{2 L}}}{d s}\right]$, where $\frac{d \frac{H_{2 N}}{H_{2 L}}}{d s}<0$ from Lemma 2. Hence, in order to know the sign of $\frac{d c_{2}}{d s}, \frac{d \frac{H_{2 N}}{H_{2 L}}}{d s}$ needs to be calculated. The indifference condition, (29), can be expressed as

$$
\left(\gamma^{\gamma} T_{2}{ }^{\alpha} T_{N}{ }^{1-\alpha}\right)^{\frac{1}{1-\gamma}}\left\{\left[(1-\alpha)\left[\delta_{N}(1-s)\right]^{\gamma}\left(\frac{H_{2 N}}{H_{2 L}}\right)^{-\alpha}\right]^{\frac{1}{1-\gamma}}-\left[\alpha\left(\delta_{L} s\right)^{\gamma}\left(\frac{H_{2 N}}{H_{2 L}}\right)^{1-\alpha}\right]^{\frac{1}{1-\gamma}}\right\}=\frac{1}{1-\gamma} \frac{\bar{l}}{\delta_{L} s} .
$$

The derivative of the LHS-RHS of (A17) with respect to $\frac{H_{2 N}}{H_{2 L}}$ equals $-\frac{1}{1-\gamma} \frac{H_{2 L}}{H_{2 N}}\left(\gamma^{\gamma} T_{2}{ }^{\alpha} T_{N}{ }^{1-\alpha}\right)^{\frac{1}{1-\gamma}}\left\{\alpha\left[(1-\alpha)\left[\delta_{N}(1-s)\right]^{\gamma}\left(\frac{H_{2 N}}{H_{2 L}}\right)^{-\alpha}\right]^{\frac{1}{1-\gamma}}+(1-\alpha)\left[\alpha\left(\delta_{L} s\right)^{\gamma}\left(\frac{H_{2 N}}{H_{2 L}}\right)^{1-\alpha}\right]^{\frac{1}{1-\gamma}}\right\}<0$. 
The derivative of the LHS-RHS of (A17) with respect to $s$ equals

$$
\begin{aligned}
& -\frac{\gamma}{1-\gamma}\left(\gamma^{\gamma} T_{2}{ }^{\alpha} T_{N}{ }^{1-\alpha}\right)^{\frac{1}{1-\gamma}}\left\{\frac{1}{1-s}\left[(1-\alpha)\left[\delta_{N}(1-s)\right]^{\gamma}\left(\frac{H_{2 N}}{H_{2 L}}\right)^{-\alpha}\right]^{\frac{1}{1-\gamma}}+\frac{1}{s}\left[\alpha\left(\delta_{L} s\right)^{\gamma}\left(\frac{H_{2 N}}{H_{2 L}}\right)^{1-\alpha}\right]^{\frac{1}{1-\gamma}}\right\}+\frac{1}{1-\gamma} \frac{\bar{l}}{\delta_{L} s^{2}} \\
& =\frac{1}{s(1-\gamma)}\left(\gamma^{\gamma} T_{2}^{\alpha} T_{N}{ }^{1-\alpha}\right)^{\frac{1}{1-\gamma}}\left\{\frac{1-\gamma-s}{1-s}\left[(1-\alpha)\left[\delta_{N}(1-s)\right]^{\gamma}\left(\frac{H_{2 N}}{H_{2 L}}\right)^{-\alpha}\right]^{\frac{1}{1-\gamma}}-\left[\alpha\left(\delta_{L} s\right)^{\gamma}\left(\frac{H_{2 N}}{H_{2 L}}\right)^{1-\alpha}\right]^{\frac{1}{1-\gamma}}\right\} .
\end{aligned}
$$

Hence,

$$
\frac{d \frac{H_{2 N}}{H_{2 L}}}{d s}=\frac{\frac{1}{s} \frac{H_{2 N}}{H_{2 L}}\left\{\frac{1-\gamma-s}{1-s}\left[(1-\alpha)\left[\delta_{N}(1-s)\right]^{\gamma}\left(\frac{H_{2 N}}{H_{2 L}}\right)^{-\alpha}\right]^{\frac{1}{1-\gamma}}-\left[\alpha\left(\delta_{L} s\right)^{\gamma}\left(\frac{H_{2 N}}{H_{2 L}}\right)^{1-\alpha}\right]^{\frac{1}{1-\gamma}}\right\}}{\alpha\left[(1-\alpha)\left[\delta_{N}(1-s)\right]^{\gamma}\left(\frac{H_{2 N}}{H_{2 L}}\right)^{-\alpha}\right]^{\frac{1}{1-\gamma}}+(1-\alpha)\left[\alpha\left(\delta_{L} s\right)^{\gamma}\left(\frac{H_{2 N}}{H_{2 L}}\right)^{1-\alpha}\right]^{\frac{1}{1-\gamma}}} .
$$

Let $B_{0} \equiv\left[(1-\alpha)\left[\delta_{N}(1-s)\right]^{\gamma}\left(\frac{H_{2 N}}{H_{2 L}}\right)^{-\alpha}\right]^{\frac{1}{1-\gamma}}$ and $B_{1} \equiv\left[\alpha\left(\delta_{L} s\right)^{\gamma}\left(\frac{H_{2 N}}{H_{2 L}}\right)^{1-\alpha}\right]^{\frac{1}{1-\gamma}}$. Using (A20), $\frac{d c_{2}}{d s}$ is proportional to

$$
\begin{aligned}
-\left[\frac{\gamma}{1-s}+\alpha\left(\frac{H_{2 N}}{H_{2 L}}\right)^{-1} \frac{d \frac{H_{2 N}}{H_{2 L}}}{d s}\right] & =-\left[\frac{\gamma}{1-s}+\frac{\frac{\alpha}{s}\left(\frac{1-\gamma-s}{1-s} B_{0}-B_{1}\right)}{\alpha B_{0}+(1-\alpha) B_{1}}\right] \\
& =-\frac{\frac{1}{s(1-s)}\left\{\alpha(1-\gamma)(1-s) B_{0}+[\gamma(1-\alpha) s-\alpha(1-s)] B_{1}\right\}}{\alpha B_{0}+(1-\alpha) B_{1}} \\
& =-\frac{\frac{1}{s(1-s)}\left[\alpha(1-\gamma)(1-s)\left(B_{0}-B_{1}\right)+\gamma(s-\alpha) B_{1}\right]}{\alpha B_{0}+(1-\alpha) B_{1}} .
\end{aligned}
$$

Since $B_{0}-B_{1}>0$ from (A17), $\frac{d c_{2}}{d s}<0$ when $s \geq \alpha$.

When $s<\alpha, \frac{d c_{2}}{d s}<(>) 0$ iff the expression inside the square bracket of (A21) is positive (negative), that is,

$$
\begin{gathered}
\alpha(1-\gamma)(1-s)\left(\left[(1-\alpha)\left[\delta_{N}(1-s)\right]^{\gamma}\left(\frac{H_{2 N}}{H_{2 L}}\right)^{-\alpha}\right]^{\frac{1}{1-\gamma}}-\left[\alpha\left(\delta_{L} s\right)^{\gamma}\left(\frac{H_{2 N}}{H_{2 L}}\right)^{1-\alpha}\right]^{\frac{1}{1-\gamma}}\right)+\gamma(s-\alpha)\left[\alpha\left(\delta_{L} s\right)^{\gamma}\left(\frac{H_{2 N}}{H_{2 L}}\right)^{1-\alpha}\right]^{\frac{1}{1-\gamma}} \\
=\left(\frac{H_{2 N}}{H_{2 L}}\right)^{\frac{1-\alpha}{1-\gamma}}\left(\alpha(1-\gamma)(1-s)\left\{\left[(1-\alpha)\left[\delta_{N}(1-s)\right]^{\gamma}\left(\frac{H_{2 N}}{H_{2 L}}\right)^{-1}\right]^{\frac{1}{1-\gamma}}-\left[\alpha\left(\delta_{L} s\right)^{\gamma}\right]^{\frac{1}{1-\gamma}}\right\}+\gamma(s-\alpha)\left[\alpha\left(\delta_{L} s\right)^{\gamma}\right]^{\frac{1}{1-\gamma}}\right)>(<) 0 \\
\Leftrightarrow \alpha(1-\gamma)(1-s)\left[(1-\alpha)\left[\delta_{N}(1-s)\right]^{\gamma}\left(\frac{H_{2 N}}{H_{2 L}}\right)^{-1}\right]^{\frac{1}{1-\gamma}}>(<)[\alpha-(\gamma(1-\alpha)+\alpha) s]\left[\alpha\left(\delta_{L} s\right)^{\gamma}\right]^{\frac{1}{1-\gamma}} \\
\Leftrightarrow \frac{H_{2 N}}{H_{2 L}}<(>) \frac{[\alpha(1-\gamma)(1-s)]^{1-\gamma}(1-\alpha)\left[\delta_{N}(1-s)\right]^{\gamma}}{\{\alpha-[\gamma(1-\alpha)+\alpha] s\}^{1-\gamma} \alpha\left(\delta_{L} s\right)^{\gamma}} .
\end{gathered}
$$

By substituting the RHS of the above equation into (A17), when $s<\alpha, \frac{d c_{2}}{d s}<(>) 0$ iff 


$$
\begin{aligned}
& \left(\gamma^{\gamma} T_{2}^{\alpha} T_{N}{ }^{1-\alpha}\right)^{\frac{1}{1-\gamma}}\left\{\begin{array}{c}
{\left[(1-\alpha)\left[\delta_{N}(1-s)\right]^{\gamma}\left(\frac{\{\alpha-[\gamma(1-\alpha)+\alpha] s\}^{1-\gamma} \alpha\left(\delta_{L} s\right)^{\gamma}}{[\alpha(1-\gamma)(1-s)]^{1-\gamma}(1-\alpha)\left[\delta_{N}(1-s)\right]^{\gamma}}\right)^{\alpha}\right]^{\frac{1}{1-\gamma}}} \\
-\left[\alpha\left(\delta_{L} s\right)^{\gamma}\left(\frac{[\alpha(1-\gamma)(1-s)]^{1-\gamma}(1-\alpha)\left[\delta_{N}(1-s)\right]^{\gamma}}{\{\alpha-[\gamma(1-\alpha)+\alpha] s\}^{1-\gamma} \alpha\left(\delta_{L} s\right)^{\gamma}}\right)^{\frac{1}{1-\gamma}}\right.
\end{array}\right\}<(>) \frac{1}{1-\gamma} \frac{\bar{l}}{\delta_{L} s} \\
& \Leftrightarrow\left(\gamma^{\gamma} T_{2}{ }^{\alpha} T_{N}{ }^{1-\alpha}\right)^{\frac{1}{1-\gamma}}(1-s)^{-\alpha}\left\{(1-\alpha)\left[\delta_{N}(1-s)\right]^{\gamma}\right\}^{\frac{1-\alpha}{1-\gamma}}\left[\alpha\left(\delta_{L} s\right)^{\gamma}\right]^{\frac{\alpha}{1-\gamma}}\left\{\frac{\alpha-[\gamma(1-\alpha)+\alpha] s}{\alpha(1-\gamma)}\right\}^{\alpha} \\
& \times\left\{1-(1-s) \frac{\alpha(1-\gamma)}{\alpha-[\gamma(1-\alpha)+\alpha] s}\right\}<(>) \frac{1}{1-\gamma} \frac{\bar{l}}{\delta_{L} s} \\
& \Leftrightarrow\left(\gamma^{\gamma} T_{2}{ }^{\alpha} T_{N}{ }^{1-\alpha}\right)^{\frac{1}{1-\gamma}}\left[(1-\alpha) \delta_{N}^{\gamma}\right]^{\frac{1-\alpha}{1-\gamma}}\left(\frac{\alpha^{\frac{\gamma}{1-\gamma}}}{1-\gamma}\right)^{\alpha} \gamma \frac{\left(\delta_{L} s\right)^{1+\alpha \frac{\gamma}{1-\gamma}}(1-s)^{(1-\alpha) \frac{\gamma}{1-\gamma}-\alpha}(\alpha-s)}{\{\alpha-[\gamma(1-\alpha)+\alpha] s\}^{1-\alpha}}<(>) \frac{\bar{l}}{1-\gamma} \text {. }
\end{aligned}
$$

If $T_{N}, T_{2}, \delta_{N}$ and $\delta_{L}$ are low enough that the LHS of (A23) at $s$ maximizing $\frac{s^{1+\alpha} \frac{\gamma}{1-\gamma}(1-s)^{(1-\alpha)} \frac{\gamma}{1-\gamma}-\alpha}{1 \alpha-s)}$ is smaller than the RHS, $\frac{d c_{2}}{d s}<0$ for any $s$; otherwise, there exist ranges of $s$ satisfying $\frac{d c_{2}}{d s}>0$.

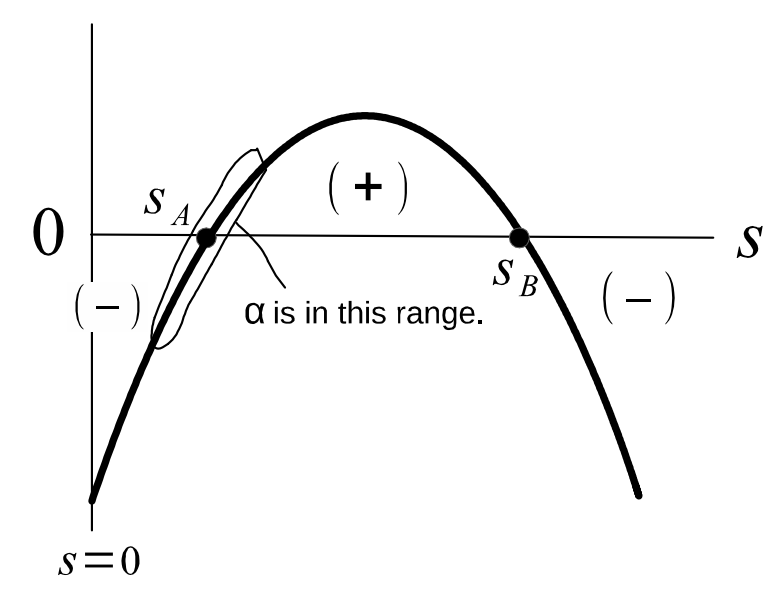

Figure A1: The shape of the quadratic function

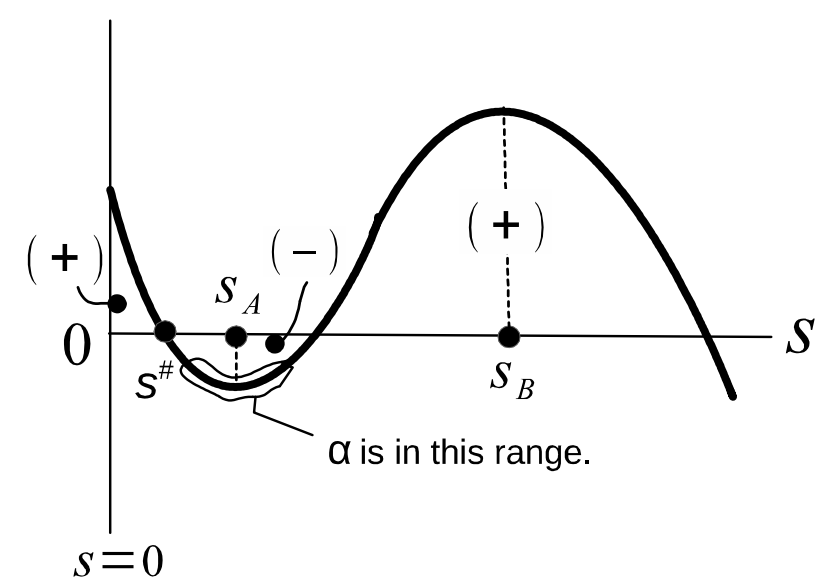

Figure A2: The shape of the cubic function 


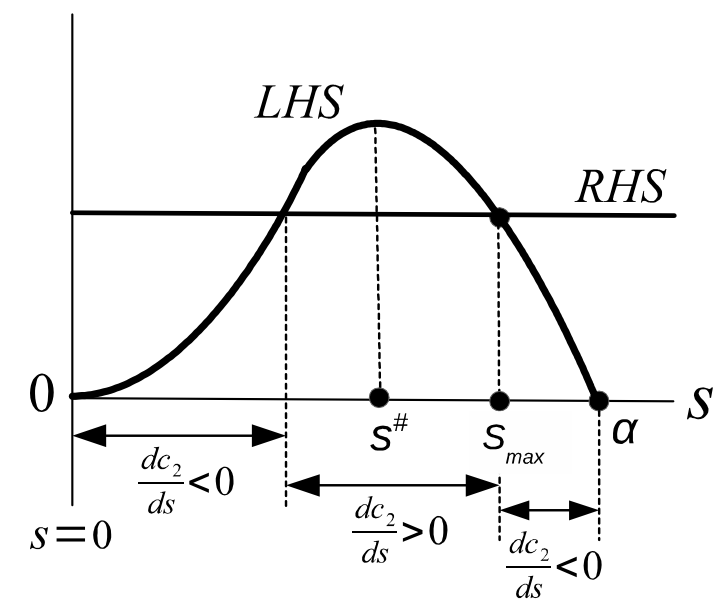

Figure A3: The determination of the sign of $\frac{d c_{2}}{d s}$ for $s<\alpha$

[Relationship between s and $c_{2}$ when $T_{N}, T_{2}, \delta_{N}$, and $\delta_{L}$ are sufficiently high] The derivative of $\frac{s^{1+\alpha} \frac{\gamma}{1-\gamma}(1-s)^{(1-\alpha)} \frac{\gamma}{1-\gamma}-\alpha}{(\alpha-s)}=\left[\frac{s^{1-\gamma(1-\alpha)}(1-s)^{\gamma-\alpha}(\alpha-s)^{1-\gamma}}{\{\alpha-[\gamma(1-\alpha)+\alpha] s\}^{1-\alpha}}\right]^{\frac{1}{1-\gamma}}$ on the LHS of (A23) with respect to $s$ equals $\frac{1}{1-\gamma}\left[\frac{s^{1-\gamma(1-\alpha)}(1-s)^{\gamma-\alpha}(\alpha-s)^{1-\gamma}}{\{\alpha-[\gamma(1-\alpha)+\alpha] s\}^{(1-\alpha)(1-\gamma)}}\right]^{\frac{1}{1-\gamma}}$ times

$$
\begin{aligned}
& {[1-\gamma(1-\alpha)] \frac{1}{s}-\frac{\gamma-\alpha}{1-s}-\frac{1-\gamma}{\alpha-s}+\frac{(1-\alpha)(1-\gamma)[\gamma(1-\alpha)+\alpha]}{\alpha-[\gamma(1-\alpha)+\alpha] s} } \\
= & \frac{[1-\gamma(1-\alpha)]\{\alpha-[\gamma(1-\alpha)+\alpha] s\}+(1-\alpha)(1-\gamma)[\gamma(1-\alpha)+\alpha] s}{\{\alpha-[\gamma(1-\alpha)+\alpha] s\} s}-\frac{(\gamma-\alpha)(\alpha-s)+(1-\gamma)(1-s)}{(1-s)(\alpha-s)} \\
= & \frac{\alpha\{[1-\gamma(1-\alpha)]-[\gamma(1-\alpha)+\alpha] s\}}{\{\alpha-[\gamma(1-\alpha)+\alpha] s\} s}-\frac{(1-\alpha)(1+\alpha-\gamma-s)}{(1-s)(\alpha-s)} \\
= & \frac{\gamma(1-\alpha)+\alpha}{\{\alpha-[\gamma(1-\alpha)+\alpha] s\} s(1-s)(\alpha-s)}\left\{\alpha\left[\frac{1-\gamma(1-\alpha)}{\gamma(1-\alpha)+\alpha}-s\right](1-s)(\alpha-s)-(1-\alpha)(1+\alpha-\gamma-s)\left[\frac{\alpha}{\gamma(1-\alpha)+\alpha}-s\right] s\right\}(\mathrm{A} 24)
\end{aligned}
$$

Let $E_{0} \equiv \frac{1-\gamma(1-\alpha)}{\gamma(1-\alpha)+\alpha}, E_{1} \equiv 1+\alpha-\gamma$, and $E_{2} \equiv \frac{\alpha}{\gamma(1-\alpha)+\alpha}$. Then, the derivative is expressed as $\frac{1}{1-\gamma}\left[\frac{s^{\alpha \gamma}(1-s)^{\gamma-\alpha}}{\{\alpha-[\gamma(1-\alpha)+\alpha] s\}^{(1-\alpha)(1-\gamma)}}\right]^{\frac{1}{1-\gamma}} \frac{\gamma(1-\alpha)+\alpha}{\{\alpha-[\gamma(1-\alpha)+\alpha] s\}(1-s)}$ times

$$
\begin{aligned}
& \alpha\left(E_{0}-s\right)(1-s)(\alpha-s)-(1-\alpha)\left(E_{1}-s\right)\left(E_{2}-s\right) s \\
= & \alpha\left[E_{0}-\left(1+E_{0}\right) s+s^{2}\right](\alpha-s)-(1-\alpha)\left[E_{1} E_{2}-\left(E_{1}+E_{2}\right) s+s^{2}\right] s \\
= & \alpha\left\{\alpha E_{0}-\left[\alpha\left(1+E_{0}\right)+E_{0}\right] s+\left(1+E_{0}+\alpha\right) s^{2}-s^{3}\right\}-(1-\alpha)\left[E_{1} E_{2} s-\left(E_{1}+E_{2}\right) s^{2}+s^{3}\right] \\
= & -s^{3}+\left[\alpha\left(1+E_{0}+\alpha\right)+(1-\alpha)\left(E_{1}+E_{2}\right)\right] s^{2}-\left\{\alpha\left[\alpha\left(1+E_{0}\right)+E_{0}\right]+(1-\alpha) E_{1} E_{2}\right\} s+\alpha^{2} E_{0} .
\end{aligned}
$$

From the first line of the above equation, the derivative is 0 at $s=0$ and negative at $s=\alpha$.

The sign of the derivative for $s \in(0, \alpha)$ can be known by examining the shape of the above cubic function. The derivative of the cubic function with respect to $s$ equals

$$
-3 s^{2}+2\left[\alpha\left(1+E_{0}+\alpha\right)+(1-\alpha)\left(E_{1}+E_{2}\right)\right] s-\left\{\alpha\left[\alpha\left(1+E_{0}\right)+E_{0}\right]+(1-\alpha) E_{1} E_{2}\right\},
$$

which is negative at $s=0$, while the sign at $s=\alpha$ is ambiguous.

The derivative of the quadratic function with respect to $s$ equals $-6 s+2\left[\alpha\left(1+E_{0}+\alpha\right)+(1-\alpha)\left(E_{1}+E_{2}\right)\right]$, which is positive at $s=0$. It is also positive at $s=\alpha$ because 


$$
\begin{aligned}
& -6 \alpha+2\left[\alpha\left(1+E_{0}+\alpha\right)+(1-\alpha)\left(E_{1}+E_{2}\right)\right] \\
= & -6 \alpha+2\left\{\alpha \frac{\alpha[\gamma(1-\alpha)+\alpha]+(1+\alpha)}{[\gamma(1-\alpha)+\alpha]}+(1-\alpha) \frac{(1+\alpha-\gamma)[\gamma(1-\alpha)+\alpha]+\alpha}{\gamma(1-\alpha)+\alpha}\right\} \\
= & -6 \alpha+2 \frac{[1-(1-\alpha) \gamma][\alpha+(1-\alpha) \gamma]+2 \alpha}{\gamma(1-\alpha)+\alpha} \\
= & 2 \frac{(1-\alpha)(1-\gamma)[3 \alpha+(1-\alpha) \gamma]}{\gamma(1-\alpha)+\alpha}>0 .
\end{aligned}
$$

Hence, both $s=0$ and $s=\alpha$ are located at the upward-sloping portion of the graph of the quadratic function. Figure A1 shows a graph of the quadratic function. Based on this figure, Figure A2 illustrates a graph of the cubic function. The sign of the derivative of $\frac{s^{1+\alpha} \frac{\gamma}{1-\gamma}(1-s)^{(1-\alpha) \frac{\gamma}{1-\gamma}-\alpha}(\alpha-s)}{\{\alpha-[\gamma(1-\alpha)+\alpha] s\}^{1-\alpha}}$ is the same as the sign of the cubic function for $s \in(0, \alpha]$ (the shapes are different because the derivative equals $\frac{1}{1-\gamma}\left[\frac{s^{\alpha \gamma}(1-s)^{\gamma-\alpha}}{\{\alpha-[\gamma(1-\alpha)+\alpha] s\}^{(1-\alpha)(1-\gamma)}}\right]^{\frac{1}{1-\gamma}} \frac{\gamma(1-\alpha)+\alpha}{\{\alpha-[\gamma(1-\alpha)+\alpha] s\}(1-s)}$ times the cubic function), while, as shown above, the sign of the derivative at $s=0$ is zero.

Therefore, there exists $s^{\sharp} \in(0, \alpha)$ such that the derivative of $\frac{s^{1+\alpha} \frac{\gamma}{1-\gamma}(1-s)^{(1-\alpha)} \frac{\gamma}{1-\gamma}-\alpha}{\{\alpha-[\gamma(1-\alpha)+\alpha] s\}^{1-\alpha}}$ equals 0 , and the derivative is 0 at $s=0$, is positive for $s \in\left(0, s^{\sharp}\right)$, and is negative for $s \in\left(s^{\sharp}, \alpha\right)$.

Based on this result, Figure A3 illustrates graphs of the LHS and the RHS of (A23) when $T_{N}$, $T_{2}, \delta_{N}$, and $\delta_{L}$ are high enough that they intersect. As shown above, when $s<\alpha, \frac{d c_{2}}{d s}<(>) 0$ iff the LHS is smaller (greater) than the RHS. Therefore, $\frac{d c_{2}}{d s}<0$ when $s$ is small, $\frac{d c_{2}}{d s}>0$ when $s$ is intermediate, and $\frac{d c_{2}}{d s}<0$ again when $s$ is large (note $\frac{d c_{2}}{d s}<0$ when $s \geq \alpha$ ).

Proof of Proposition 1. (i) From Lemma 3 (i), net earnings and consumption of group 2 individuals decrease with $s$ when $e_{2 L}^{*}=0$. From (ii) of the lemma, if $T_{N}, T_{2}, \delta_{N}$, and $\delta_{L}$ are low enough, they decrease with $s$ when $e_{2 L}^{*}>0$ too and thus they decrease with $s$ for any $s$. Even when $T_{N}, T_{2}, \delta_{N}$, and $\delta_{L}$ are high enough that net earnings and consumption increase with $s$ for intermediate $s$ when $e_{2 L}^{*}>0$ (Lemma 3 (ii)), they decrease with $s$ for any $s$, if such range of $s$ is not effective, i.e. $e_{2 L}^{*}>0$ is not true. From (A23) in the proof of Lemma 3, the supremum of $s$ satisfying $\frac{d c_{2}}{d s}>0$, which is $s_{\max }$ in Figure A3 and is smaller than $\alpha$, increases with $T_{N}, T_{2}, \delta_{N}$, and $\delta_{L}$. From Lemma $1, e_{2 L}^{*}=0$ iff $s \leq \underline{s}$ and $s \geq \bar{s}(\underline{s}<1-\gamma(1-\alpha)<\bar{s})$, where $\underline{s}$ decreases ( $\bar{s}$ increases) with $T_{N}, T_{2}, \delta_{N}$, and $\delta_{L}$. Hence, if these exogenous variables are low enough that $s_{\max } \leq \underline{s}$, consumption and net earnings decrease with $s$ for any $s$.

(ii) Only the proof of the result on the consumption is presented, because net earnings in unit of the final good equal consumption minus wealth. From the proof of (i), if $T_{N}, T_{2}, \delta_{N}$, and $\delta_{L}$ are high enough that $s_{\max }>\underline{s}$, consumption increases with $s$ when $e_{2 L}^{*}>0$ and $s$ is intermediate. From Lemma $3, c_{2}$ decreases with $s$ for small $s$, increases with $s$ for intermediate $s$, and decreases with $s$ for large $s$, where, from Lemmas 1 and $3, c_{2}$ decreases with $s$ at least for $s \leq \underline{s}$ and $s \geq \min \{\alpha, \bar{s}\}$.

Hence, $c_{2}$ is maximized either at $s=s_{\max }<\alpha$ or at $s=0$. From (30), $c_{2}$ at an intermediate $s$ is greater (smaller) than $c_{2}$ at $s=0$, where $e_{2 L}^{*}=0$, iff 


$$
\begin{gathered}
(1-s)^{\gamma}\left(\frac{H_{2 N}}{H_{2 L}} \mid \text { intermediate } s\right)^{-\alpha}>(<)\left(\left.\frac{H_{2 N}}{H_{2 L}}\right|_{s=0}\right)^{-\alpha} \\
\Leftrightarrow(1-s)^{\gamma}\left(\left.\frac{H_{2 N}}{H_{2 L}}\right|_{\text {intermediate } s}\right)^{-\alpha}>(<)\left\{\left(\frac{1-\alpha}{\alpha} \frac{1-\gamma}{(\bar{l})^{\gamma}}\right)^{1-\gamma}\left[(1-\alpha) \gamma \delta_{N} T_{2}^{\alpha} T_{N}{ }^{1-\alpha}\right]^{\gamma}\right\}^{-\frac{\alpha}{1-\gamma(1-\alpha)}} \\
\left.\Leftrightarrow \frac{H_{2 N}}{H_{2 L}}\right|_{\text {intermediate } s}<(>)(1-s)^{\frac{\gamma}{\alpha}}\left\{\left(\frac{1-\alpha}{\alpha} \frac{1-\gamma}{(\bar{l})^{\gamma}}\right)^{1-\gamma}\left[(1-\alpha) \gamma \delta_{N} T_{2}{ }^{\alpha} T_{N}{ }^{1-\alpha}\right]^{\gamma}\right\}^{\frac{1}{1-\gamma(1-\alpha)}}
\end{gathered}
$$

From (A22) in the proof of Lemma 3,s satisfies $\frac{d c_{2}}{d s}=0$ and thus could be equal to $s_{\max }$ iff $\frac{H_{2 N}}{H_{2 L}}=\frac{[\alpha(1-\gamma)(1-s)]^{1-\gamma}(1-\alpha)\left[\delta_{N}(1-s)\right]^{\gamma}}{\{\alpha-[\gamma(1-\alpha)+\alpha] s\}^{1-\gamma} \alpha\left(\delta_{L} s\right)^{\gamma}}$. Hence, from this equation and (A26), the condition for $c_{2}$ at $s=s_{\max }$ to be greater (smaller) than $c_{2}$ at $s=0$ is given by

$$
\begin{gathered}
(1-s)^{\frac{\gamma}{\alpha}}\left\{\left(\frac{1-\alpha}{\alpha} \frac{1-\gamma}{(\bar{l})^{\gamma}}\right)^{1-\gamma}\left[(1-\alpha) \gamma \delta_{N} T_{2}{ }^{\alpha} T_{N}{ }^{1-\alpha}\right]^{\gamma}\right\}^{\frac{1}{1-\gamma(1-\alpha)}}>(<) \frac{[\alpha(1-\gamma)(1-s)]^{1-\gamma}(1-\alpha)\left[\delta_{N}(1-s)\right]^{\gamma}}{\{\alpha-[\gamma(1-\alpha)+\alpha] s\}^{1-\gamma} \alpha\left(\delta_{L} s\right)^{\gamma}} \\
\Leftrightarrow \alpha s^{\gamma}(1-s)^{\frac{\gamma(1-\alpha)}{\alpha}}\left\{\left(\frac{1-\alpha}{\alpha} \frac{1-\gamma}{(\bar{l})^{\gamma}}\right)^{1-\gamma}\left[(1-\alpha) \gamma\left(\delta_{N}\right)^{\gamma(1-\alpha)}\left(\delta_{L}\right)^{1-\gamma(1-\alpha)} T_{2}^{\alpha} T_{N}{ }^{1-\alpha}\right]^{\gamma}\right\}^{\frac{1}{1-\gamma(1-\alpha)}}>(<)(1-\gamma)^{1-\gamma}(1-\alpha)\left\{\frac{\alpha(1-s)}{\alpha-[\gamma(1-\alpha)+\alpha] s}\right\}^{1-\gamma}
\end{gathered}
$$

Hence, if $T_{N}, T_{2}, \delta_{N}$, and $\delta_{L}$ are is sufficiently large, $c_{2}$ at $s=s_{\max }$ is greater than $c_{2}$ at $s=0$, otherwise $c_{2}$ at $s=0$ is greater. From Figure A3 of the proof of Lemma 3 (ii), $s_{\max }$ is the largest of two values of $s$ at which the LHS and the RHS of (A23) are equal. As $T_{N}, T_{2}, \delta_{N}$ and $\delta_{L}$ become higher, the graph of the LHS shifts upward and thus $s_{\max }$ increases.

Proof of Proposition 2. (i) From Lemma $1, h_{2 L}^{*}$ is lowest when $s \leq \underline{s}$ and $s \geq \bar{s}$. When $s \in(\underline{s}, \bar{s})$ and thus $e_{2 L}^{*}>0$, from (13) and (24),

$$
\begin{aligned}
\frac{d h_{2 L}^{*}}{d s} & \propto \frac{1}{s}+(1-\alpha)\left(\frac{H_{2 N}}{H_{2 L}}\right)^{-1} \frac{d \frac{H_{2 N}}{H_{2 L}}}{d s} \\
& \left.=\frac{1}{s}\left[1+\frac{(1-\alpha)\left(\frac{1-\gamma-s}{1-s} B_{0}-B_{1}\right)}{\alpha B_{0}+(1-\alpha) B_{1}}\right] \text { (from (A20) in the proof of Lemma } 3\right) \\
& =\frac{1}{s} \frac{\alpha B_{0}+(1-\alpha) \frac{1-\gamma-s}{1-s} B_{0}}{\alpha B_{0}+(1-\alpha) B_{1}} \\
& =\frac{1}{s(1-s)} \frac{(1-s)-(1-\alpha) \gamma}{\alpha B_{0}+(1-\alpha) B_{1}} B_{0}>(<) 0 \text { for } s<(>) 1-(1-\alpha) \gamma, \\
\text { where } B_{0} & \equiv\left[(1-\alpha)\left[\delta_{N}(1-s)\right]^{\gamma}\left(\frac{H_{2 N}}{H_{2 L}}\right)^{-\alpha}\right]^{\frac{1}{1-\gamma}} \text { and } B_{1} \equiv\left[\alpha\left(\delta_{L} s\right)^{\gamma}\left(\frac{H_{2 N}}{H_{2 L}}\right)^{1-\alpha}\right]^{\frac{1}{1-\gamma}} .
\end{aligned}
$$

Hence, $h_{2 L}^{*}$ is maximized at $s=1-(1-\alpha) \gamma \in(\underline{s}, \bar{s})$.

(ii) From (12), $\frac{d h_{2 N}^{*}}{d s}$ is proportional to $-\left[\frac{1}{1-s}+\alpha\left(\frac{H_{2 N}}{H_{2 L}}\right)^{-1} \frac{d \frac{H_{2 N}}{H_{2 L}}}{d s}\right]$, while from the proof of Lemma $3, \frac{d c_{2}}{d s}$ is proportional to $-\left[\frac{\gamma}{1-s}+\alpha\left(\frac{H_{2 N}}{H_{2 L}}\right)^{-1} \frac{d \frac{H_{2 N}}{H_{2 L}}}{d s}\right]$. When $s \leq \underline{s}$ and $s \geq \bar{s}$ and thus $e_{2 L}^{*}=0, c_{2}$ decreases with $s$ from the lemma. Hence, $h_{2 N}^{*}$ decreases with $s$ as well.

When $s \in(\underline{s}, \bar{s})$, 


$$
\begin{aligned}
\frac{d h_{2 N}^{*}}{d s} & \propto-\left[\frac{1}{1-s}+\alpha\left(\frac{H_{2 N}}{H_{2 L}}\right)^{-1} \frac{d \frac{H_{2 N}}{H_{2 L}}}{d s}\right]=-\left[\frac{1}{1-s}+\frac{\frac{\alpha}{s}\left(\frac{1-\gamma-s}{1-s} B_{0}-B_{1}\right)}{\alpha B_{0}+(1-\alpha) B_{1}}\right](\text { from }(\mathrm{A} 20)) \\
& =-\frac{\frac{1}{s(1-s)}\left[\alpha(1-\gamma) B_{0}+(s-\alpha) B_{1}\right]}{\alpha B_{0}+(1-\alpha) B_{1}}<-\frac{\frac{1}{s(1-s)} B_{1}(s-\alpha \gamma)}{\alpha B_{0}+(1-\alpha) B_{1}}\left(\text { since } B_{0}>B_{1}\right) .
\end{aligned}
$$

Thus, $\frac{d h_{2 N}^{*}}{d s}<0$ at least for $s \geq \min \{\alpha \gamma, \bar{s}\}$. The rest of the result can be proved similarly to the corresponding proof on consumption of Lemma 3 (ii) and Proposition 1 (ii).

From (12), (21), and (22),

$\left(h_{2 N}^{*}\right.$ at $\left.s=0\right)>(<)\left(h_{2 N}^{*}\right.$ at intermediate $\left.s\right) \Leftrightarrow($ net wages at $s=0)>(<)($ net wages intermediate $s)$

$$
\Leftrightarrow\left(\left.\frac{H_{2 N}}{H_{2 L}}\right|_{s=0}\right)^{-\alpha}>(<)(1-s)\left(\left.\frac{H_{2 N}}{H_{2 L}}\right|_{\text {intermediate } s}\right)^{-\alpha} \text {. }
$$

Then, the result that $h_{2 N}^{*}$ is maximized at intermediate $s($ at $s=0)$ when $T_{N}, T_{2}, \delta_{N}$, and $\delta_{L}$ are sufficiently high (low) can be proved similarly to the corresponding proof on consumption of Proposition 1 (ii).

Proof of $e_{2 N}^{*}>e_{2 L}^{*}$. From (21) and (24),

$$
\begin{aligned}
e_{2 N}^{*} & =\left[(1-\alpha) T_{2}^{\alpha} T_{N}{ }^{1-\alpha} \gamma\left(\delta_{N}(1-s)\right)^{\gamma}\left(\frac{H_{2 N}}{H_{2 L}}\right)^{-\alpha}\right]^{\frac{1}{1-\gamma}}>e_{2 L}^{*}=\frac{1}{\delta_{L} s}\left\{\left[\alpha \gamma \delta_{L} s T_{2}^{\alpha} T_{N}{ }^{1-\alpha}\left(\frac{H_{2 N}}{H_{2 L}}\right)^{1-\alpha}\right]^{\frac{1}{1-\gamma}}-\bar{l}\right\} \\
& \Leftrightarrow\left[\gamma T_{2}^{\alpha} T_{N}{ }^{1-\alpha}\left(\frac{H_{2 N}}{H_{2 L}}\right)^{-\alpha}\right]^{\frac{1}{1-\gamma}} \delta_{L} s\left\{\left[(1-\alpha)\left(\delta_{N}(1-s)\right)^{\gamma}\right]^{\frac{1}{1-\gamma}}-\left[\alpha\left(\delta_{L} s\right)^{\gamma} \frac{H_{2 N}}{H_{2 L}}\right]^{\frac{1}{1-\gamma}}\right\}+\bar{l}>0 . \quad \text { (A30) }
\end{aligned}
$$

Thus, $e_{2 N}^{*}>e_{2 L}^{*}$ if $(1-\alpha)\left(\delta_{N}(1-s)\right)^{\gamma} \geq \alpha\left(\delta_{L} s\right)^{\gamma} \frac{H_{2 N}}{H_{2 L}}$.

Because the indifference condition, (29), can be expressed as

$$
\left[\gamma^{\gamma} T_{2}{ }^{\alpha} T_{N}{ }^{1-\alpha}\left(\frac{H_{2 N}}{H_{2 L}}\right)^{-\alpha}\right]^{\frac{1}{1-\gamma}}\left(\left\{(1-\alpha)\left[\delta_{N}(1-s)\right]^{\gamma}\right\}^{\frac{1}{1-\gamma}}-\left[\alpha\left(\delta_{L} s\right)^{\gamma}\left(\frac{H_{2 N}}{H_{2 L}}\right)\right]^{\frac{1}{1-\gamma}}\right)=\frac{1}{1-\gamma} \frac{\bar{l}}{\delta_{L} s}
$$

$(1-\alpha)\left[\delta_{N}(1-s)\right]^{\gamma}>\alpha\left(\delta_{L} s\right)^{\gamma} \frac{H_{2 N}}{H_{2 L}}$ must hold. Therefore, $e_{2 N}^{*}>e_{2 L}^{*}$ is always true. 